\title{
Digital twin: a state-of-the-art review of its enabling technologies, applications and challenges
}

\author{
Weifei $\mathrm{Hu}$ \\ State Key Laboratory of Fluid Power and Mechatronic Systems, \\ Zhejiang University, Hangzhou, P.R. China and \\ School of Mechanical Engineering, Zhejiang University, \\ Hangzhou, P.R. China \\ Tongzhou Zhang and Xiaoyu Deng \\ School of Mechanical Engineering, Zhejiang University, \\ Hangzhou, P.R. China, and \\ Zhenyu Liu and Jianrong Tan \\ State Key Laboratory of Fluid Power and Mechatronic Systems, \\ Zhejiang University, Hangzhou, P.R. China and \\ School of Mechanical Engineering, Zhejiang University, \\ Hangzhou, P.R. China
}

\begin{abstract}
Digital twin (DT) is an emerging technology that enables sophisticated interaction between physical objects and their virtual replicas. Although DT has recently gained significant attraction in both industry and academia, there is no systematic understanding of DT from its development history to its different concepts and applications in disparate disciplines. The majority of DT literature focuses on the conceptual development of DT frameworks for a specific implementation area. Hence, this paper provides a state-of-the-art review of DT history, different definitions and models, and six types of key enabling technologies. The review also provides a comprehensive survey of DT applications from two perspectives: (1) applications in four product-lifecycle phases, i.e. product design, manufacturing, operation and maintenance, and recycling and (2) applications in four categorized engineering fields, including aerospace engineering, tunneling and underground engineering, wind engineering and Internet of things (IoT) applications. DT frameworks, characteristic components, key technologies and specific applications are extracted for each DT category in this paper. A comprehensive survey of the DT references reveals the following findings: (1) The majority of existing DT models only involve one-way data transfer from physical entities to virtual models and (2) There is a lack of consideration of the environmental coupling, which results in the inaccurate representation of the virtual components in existing DT models. Thus, this paper highlights the role of environmental factor in DT enabling technologies and in categorized engineering applications. In addition, the review discusses the key challenges and provides future work for constructing DTs of complex engineering systems.
\end{abstract}

Keywords Digital twin, Enabling technologies, Virtual modeling, Environmental coupling, Cyber-physical system, Design engineering, Internet of things

Paper type Book review

(C) Weifei Hu, Tongzhou Zhang, Xiaoyu Deng, Zhenyu Liu and Jianrong Tan. Published in Journal of Intelligent Manufacturing and Special Equipment. Published by Emerald Publishing Limited. This article is published under the Creative Commons Attribution (CC BY 4.0) licence. Anyone may reproduce, distribute, translate and create derivative works of this article (for both commercial and noncommercial purposes), subject to full attribution to the original publication and authors. The full terms of this licence may be seen at http://creativecommons.org/licences/by/4.0/legalcode

We gratefully acknowledge the funding support from the National Key R\&D Program of China (grant numbers 2019YFB1705200) and the National Natural Science Foundation of China (NSFC) (grant numbers 51905475) and Key Research and Development Program of Zhejiang Province (grant No. 2021C01008). 


\section{JIMSE 2,1}

\section{2}

\section{Introduction}

To trigger the core value of the Internet of things (IoT), stakeholders in both industry and academia are merging a large number of physical entities with virtual models, which is considered one of the most important aspects of the digital twin (DT). ADT is a real-time digital replica of a physical entity, in which the real part can be mirrored in a virtual environment and continuously updated from multiple sources for various purposes (Fuller et al., 2019).

As an emerging technology, the development of DT matches the profound strategic plans of some leading manufacturing countries. For example, in accordance with the development and application of a cyber-physical system (CPS), DT has become a key component in the Made in China 2025 plan, which targets the development of intelligent control systems; industrial application software, fault diagnosis software and related tools; and sensor and communication system protocols to realize real-time connection, accurate identification, effective interaction, and intelligent control of manufacturing equipment and products (State Council Of China, 2015). Industry 4.0, which is a German high-tech strategy, requires the CPS as one of the four major components to predict and optimize a production system in real time (Negri et al., 2017). This requirement aligns with the targets of implementing DT in a manufacturing system.

DT will contribute considerable economic benefit; Market Research Future predicts that the DT market will reach 35 billion USD by 2025 (Market Research Future, 2019). A joint report by the Fraunhofer Institute and the industry association Bitkom indicated that the German gross value of the DT market can be boosted by a cumulative 267 billion euros by 2025 after introducing Industry 4.0 (Lee et al., 2015). DT has also recently gained an increasing amount of attention in academia. According to statistical data of search results from Google Scholar (2020), Figure 1 shows the number of DT-related studies that were published from 2011 to 2019. The surge of DT research is clearly seen from 2017-2019; Gartner listed DT as one of the top 10 technological trends with strategic value during these 3 years (Panetta, 2016, 2017, 2018).

DT has been extensively investigated in different industry fields. DT allows companies to have a virtual copy of their product in their full lifecycles, rapidly detect defaults, solve physical

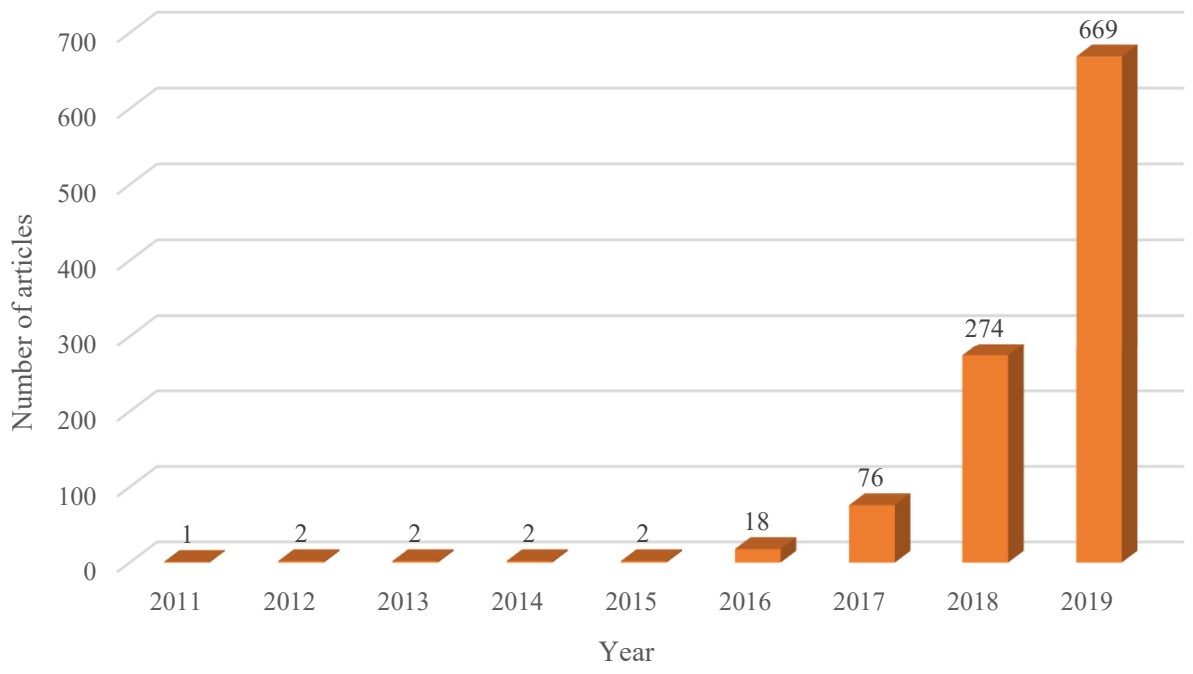

Source(s): Google Scholar (2020)
Figure 1.

Number of published articles about DT 
issues sooner, more accurately predict outcomes and better serve their customers (Parrott and Warshaw, 2017). Tuegel (2012) proposed an airframe digital twin (ADT) that was specified for spacecraft. Tao and Zhang (2017) established DT shop-floor (DTS) technology for intellectual shop-floor design. General Electric (GE) has developed the platform "Predix" for a DT wind farm (Noel and Jackson, 2015). A DT for healthcare and a DT for smart cities is categorized in Fuller et al. (2019) and Qi et al. (2021), respectively. Although it has recently gained significant attraction in both industry and academia, there is no systematic understanding of DT from its development history to different concepts and applications in disparate disciplines. The majority of DT literature focuses on the conceptual development of DT frameworks for a specific implementation area. However, a unified, general and abstract theoretical framework and practical platform of DT has not appeared (Negri et al., 2017). Thus, it is necessary to provide a systematic summary of DT history, definitions, models, technologies and applications to promote the maturity of DT in an extensive range of engineering fields.

Based on a comprehensive survey of DT literature, this paper provides a state-of-the-art review of DT history, different definitions and models, as well as 6 types of key enabling technologies according to the 6-dimensional DT model, which is based on 5-dimensional DT model proposed in Tao and Zhang (2017), while concerning environment factor simultaneously. The review also categorizes the various DT applications from two perspectives (1) applications in four product-lifecycle phases (i.e. product design, manufacturing, operation and maintenance and recycling) and (2) applications in four engineering fields, including aerospace engineering, tunneling and underground engineering, wind engineering, and IoT applications. Another contribution of this paper is that environmental coupling technologies are highlighted and summarized for creating highfidelity virtual components in DT.

The remainder of the paper is organized as follows: Section 2 summarizes the history, definitions and models of DT. Section 3 summarizes the key technologies required for each of the components of the five-dimensional DT model. Section 4 elaborates DT technologies and the applications from the perspective of the four production lifecycle phases and four different engineering applications. The key challenges of constructing DT are extracted in Section 5, followed by concluding remarks in Section 6 .

\section{History, definitions and models}

\subsection{DT history}

The concept of the "twin" dates to the National Aeronautics and Space Administration (NASA) Apollo program in the 1970s, where a replica of space vehicles on Earth was built to mirror the condition of the equipment during the mission (Rosen et al., 2015; Miskinis, 2019). This replica was the first application of the "twin" concept. In 2003, DT was proposed by Michael Grieves in his product lifecycle management (PLM) course as "virtual digital representation equivalent to physical products" (Grieves, 2014). In 2012, DT was applied by NASA to integrate ultra-high-fidelity simulation with a vehicle's on-board integrated vehicle health management system, maintenance history, and all available historical and fleet data to mirror the life of its flying twin and enable unprecedented levels of safety and reliability (Glaessgen and Stargel, 2012; Tuegel et al., 2011a). The advent of IoT boosts the development of DT technology in the manufacturing industry. Enterprises such as Siemens and GE developed platforms of DT for real-time monitoring, inspection and maintenance (Eliane Fourgeau, 2016). Recently, Tao and Zhang (2017) proposed a five-dimensional DTS framework, which provides theoretical guidance for the digitalization and intellectualization of the manufacturing industry. From 2017 to 2019, DT has been continuously selected as one of the top 10 technological trends with strategic values by Gartner (Panetta, 2016, 2017, 2018). The history of the DT is briefly summarized in Figure 2. 


\section{JIMSE 2,1}

4

Figure 2.

Brief history of DT

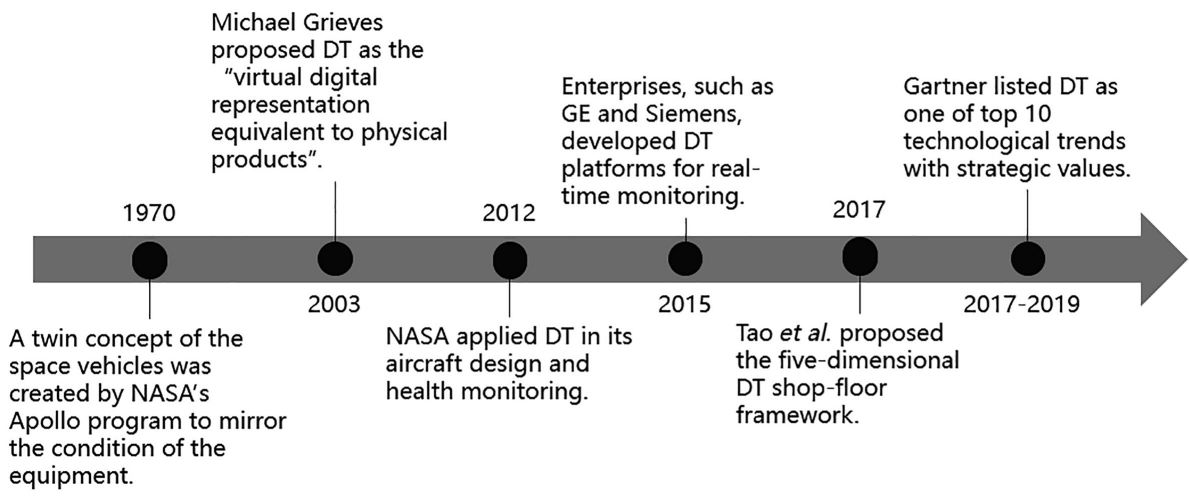

\subsection{DT definitions}

The definitions of DT have been continuously evolving, as DT-enabling technologies (e.g. sensing technology, modeling technology, data management method, DT service technology and data connection technology, Qi et al., 2021) have been developed since the 2000s. Although the concept of DT was proposed by Michael Grieves as the "virtual digital representation equivalent to physical products" in 2003 (Grieves, 2014), the development of DT was stagnant until 2012, when NASA defined DT as an integrated multi-physics, multi-scale, probabilistic simulation of an as-built vehicle or system that employs the best available physical models, sensor updates, fleet history, etc., to mirror the life of its corresponding flying twin (Glaessgen and Stargel, 2012). Subsequently, the aerospace field became an important research branch of the DT. In 2015, Ríos et al. (2015) substituted "vehicle" with "product", which extended the definition of DT for more general purposes. The DT has been defined specifically in many industrial fields. For instance Tao et al. (2019), considered DT "a real mapping of all components in the product lifecycle using physical data, virtual data and interaction data between them" in product design engineering. In IoT engineering, DT is defined as an evolving digital profile of the historical and current behavior of a physical object or process that helps optimize business performance (Parrott and Warshaw, 2017).

Table 1 lists various DT definitions and their corresponding reference and applied fields in chronological order. These definitions reveal the following points:

(1) The basic components of DT, including the physical entity, virtual model, and connection, are included, either explicitly or implicitly.

(2) Different definitions have varied highlighted aspects raised by different people. For example, Kritzinger et al. (2018) focus on data transmission, while (Liau et al., 2017) concentrate on services of DT.

It is challenging to use a common DT definition for all industrial fields. In addition, the influence from the virtual model to the physical entity is not mentioned in some DT definitions (e.g. Liau et al., 2017; Vrabic et al., 2018; Grieves and Vickers, 2017; Madni et al., 2019). According to Kritzinger et al. (2018), the definition in Madni et al. (2019) is more consistent with the description of the digital shadow (DS), while the definitions of Vrabic et al. (2018) and Grieves and Vickers (2017) are more consistent with the requirements of the digital model (DM). Although existing DT definitions may highlight specific aspects or components of DT systems, a general definition of DT may refer to the digital replica of physical assets, processes, people, places and systems, which provides both the elements and the dynamics of how the complex system operates and evolves throughout its lifecycle. 


\begin{tabular}{|c|c|c|c|}
\hline References & Definitions & Applied fields & \\
\hline Grieves (2014) & $\begin{array}{l}\text { The virtual digital representation equivalent to physical } \\
\text { products }\end{array}$ & General & \\
\hline Glaessgen and & The DT is an integrated multiphysics, multiscale, probabilistic & Aerospace & \\
\hline Stargel (2012) & $\begin{array}{l}\text { simulation of an as-built vehicle or system that employs the best } \\
\text { available physical models, sensor updates, fleet history, etc., to } \\
\text { mirror the life of its corresponding flying twin }\end{array}$ & engineering & 5 \\
\hline $\begin{array}{l}\text { Hochhalter et al. } \\
\text { (2014) }\end{array}$ & $\begin{array}{l}\text { The DT is a life management and certification paradigm, where } \\
\text { model and simulations consist of as-built vehicle states, as- } \\
\text { experienced loads and environments, and other vehicle-specific } \\
\text { history to enable high-fidelity modeling of individual aerospace } \\
\text { vehicles throughout their service lives }\end{array}$ & $\begin{array}{l}\text { Aerospace } \\
\text { engineering }\end{array}$ & \\
\hline Ríos et al. (2015) & $\begin{array}{l}\text { An integrated multiphysics, multiscale, probabilistic simulation } \\
\text { of an as-built product that employs the best available physical } \\
\text { models, sensor updates, history data, etc., to mirror the life of its } \\
\text { corresponding physical twin }\end{array}$ & General & \\
\hline Parrott and & An evolving digital profile of the historical and current behavior & IoT & \\
\hline Warshaw (2017) & $\begin{array}{l}\text { of a physical object or process that helps optimize business } \\
\text { performance }\end{array}$ & & \\
\hline Liu et al. (2018) & $\begin{array}{l}\text { A replication of real physical production system in the DM, } \\
\text { which are utilized for system optimization, monitoring, } \\
\text { diagnostics and prognostics via integration of artificial } \\
\text { intelligence, machine learning and software analytics with large } \\
\text { volumes of data from physical systems }\end{array}$ & $\begin{array}{l}\text { Manufacture } \\
\text { engineering }\end{array}$ & \\
\hline Vrabic et al. (2018) & $\begin{array}{l}\text { The DT is a digital representation of a physical item or } \\
\text { assembly that uses integrated simulations and service data }\end{array}$ & General & \\
\hline Tao et al. (2019) & $\begin{array}{l}\text { A real mapping of all components in the product lifecycle using } \\
\text { physical data, virtual data and interaction data between them }\end{array}$ & Design engineering & \\
\hline $\begin{array}{l}\text { Grieves and Vickers } \\
(2017)\end{array}$ & $\begin{array}{l}\text { A set of virtual information constructs that fully describes a } \\
\text { potential or actual physical manufacturing product from the } \\
\text { micro atomic level to the macro geometrical level }\end{array}$ & General & \\
\hline Madni et al. (2019) & $\begin{array}{l}\text { A virtual instance of a physical system (twin) that is continually } \\
\text { updated with the physical system's performance, maintenance, } \\
\text { and health status data throughout the physical system's } \\
\text { lifecycle }\end{array}$ & General & $\begin{array}{r}\text { Table 1. } \\
\text { Different definitions of } \\
\text { DT in the literature }\end{array}$ \\
\hline
\end{tabular}

\subsection{DT models}

Associated with the varied DT definitions, various DT models (or frameworks) have been raised for different engineering fields. The early DT model proposed by Grieves (2014) consists of three components: physical product, virtual product and their connection. The virtual product contains not only geometrical information but also behavioral characteristics that show the system performance in response to external stimuli. The NASA and US Air Force Research Laboratory have applied a DT framework to their aircrafts to achieve a more efficient design, greater ability of aircraft, reduction in unexpected cracks and better structural inspection (Tuegel et al., 2011a; Gockel et al., 2012).

Based on the original 3D model of DT, Qi et al. (2021) and Tao and Zhang (2017) proposed a five-dimensional DTS model for intellectual shop-floor design, including the physical entities, virtual models, services, DT data and connections. While in reconfigurable manufacturing, Zhang et al. (2019b) proposed the reconfigurable digital twin (RDT) model, including the geometry, physics, capability, behavior and rule. The DTS model and RDT model are specified for shop-floor configuration to quickly implement CPS in smart manufacturing.

Other DT models, e.g. DT for waste electrical and electronic equipment (WEEE) (Wang and Wang, 2019), DT-enabled fault diagnosis framework (Tao et al., 2018d) and DT-driven 


\section{JIMSE 2,1}

6

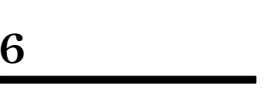

Tao and Zhang

(2017)

Zhang et al. (2019c)

Gockel et al. (2012)

Qi et al. (2021) Five-dimensional DT model

Zhang et al. (2019b) Reconfigurable digital twin

Wang and Wang

(2019)

Wang et al. (2019)

Table 2.

Tao et al. (2019)

Summary of different

DT models
Digital twin shop-floor (DTS)

Product manufacturing digital twin (PMDT) (RDT)

\section{Original DT}

Airframe digital twin (ADT)

Digital twin for waste electrical and electronic equipment (WEEE)

Digital twin enabled fault diagnosis framework

DT-driven product design

(DTPD) framework
Key components

Physical products, virtual products, and connection between physical and virtual products

Structural definition, structural models, material state evolution models, and flight dynamics

Physical entities, virtual models, services, DT data, and connections

Physical shop-floor, virtual shop-floor, shop-floor services, shop-floor DT data, and connection

Product definition model (PDM), geometric and

shape model (GSM), manufacturing attribute model (MAM), behavior and rule model (BRM), and data fusion model (DFM)

Physical layer, model layer, data layer and service layer

Cyber world, service flow, DT knowledge, and physical flow

Physical system, enabling technology, DT model, and predictive maintenance

Planning and task clarification, conceptual design, embodiment design, detail design, virtual verification

product design (DTPD) framework (Tao et al., 2019) are referenced in the corresponding literature. Table 2 provides a concise summary of various DT models or frameworks, their key components and the corresponding references.

\section{DT enabling technologies}

To construct DT, a variety of enabling technologies should be implemented, as demonstrated in Figure 3. Tao and Zhang (2017) has already proposed a five-dimensional model which has encompassed comprehensive elements required for DT. Based on the five-dimensional DT model, we further emphasize the environmental coupling technologies when constructing a high-fidelity DT model. This extended DT model is particularly suitable for health-condition monitoring of complex equipment in varied engineering fields, e.g. in tunneling and underground space engineering, marine engineering and wind engineering.

The physical object ought to be capable to percept the outside world. Thus, sensing technology needs implementation to gain a full understanding of the environment. Once data are acquired, the virtual model should then be adjusted to keep track of the changes in physical entities. To replicate the physical world as realistically as possible, the virtual model should contain an intact features set that consists of geometrical, physical, behavioral and rule information (Tao and Zhang, 2017; Qi et al., 2021; Tao et al., 2018d). Since massive multisource heterogeneous data are generated during the operation of a physical object, big data analytics technologies are required to collect, transmit, store and process the data. DT services cope with the concrete functions depending on the usage of the physical object. To deliver the original and processed data, data transmission technologies, such as different communication protocols and IoT technologies, should be imported. Data-driven technology is also essential for controlling the physical object to respond to the commands from the upper level. The environment factor is an important component of DT, which provides information necessary to secure the consistency of physical entity and virtual model, collect and integrate information on all elements, precisely predict the change of the environment. Thus, the 


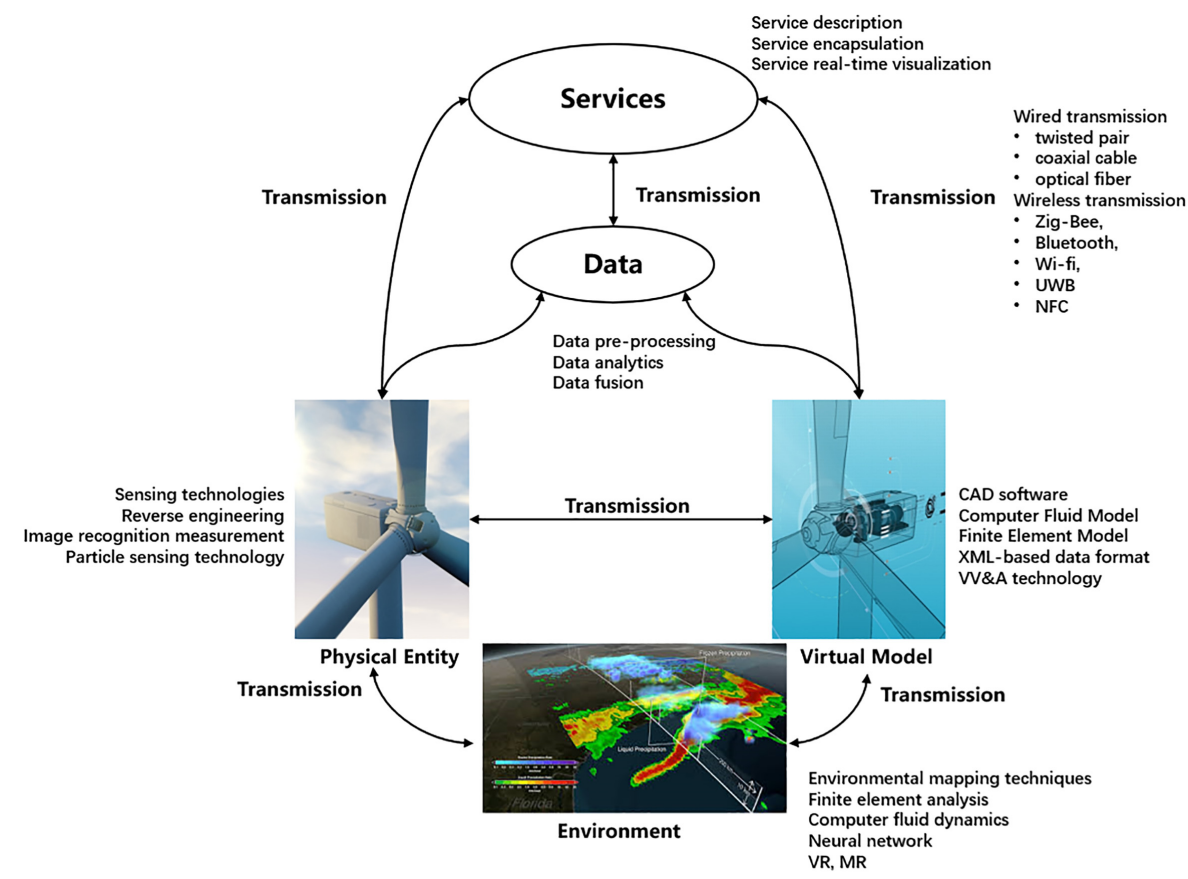

Book review

environment coupling technology is required in order to consider the effect of environmental factors. This section surveys the enabling technologies for constructing a DT model.

\subsection{Technologies for physical objects}

Physical objects are key components of the DT because they are the sources of massive multisource heterogeneous data from the real world. To perceive real-world data, such as geometric shape, physical property and mechanical precision, sensing and measurement technologies are implemented for DT, including IoT sensing technologies, reverse engineering, image recognition measurement and particle sensing technology, etc. (Qi et al., 2021). In aerospace engineering, Hochhalter et al. (2014) implemented sensory material, which produces a phase transition when sufficient strain occurs, into structural alloy to improve the reliability of crack detection.

Because physical objects are used to performing designated tasks, the control technologies of the physical objects need to be considered when implementing the DT, including the power selection (e.g. electrical power and hydraulic power), mechanical transmission design (e.g. gear drive, belt drive, and connecting rod drive) and control technologies (e.g. programmable control, supervisory control, simulation-based control, etc.) (Qi et al., 2021; Zhuang et al., 2018). For control technologies, Wang et al., (2017) utilized the IEC-61499-based function block to compile the algorithm into robot control codes. Atorf et al. (2017) controlled the robot arm of an assembly line in a simulation-based manner, where users can implement complex abort conditions and objective functions to control the automated simulations.

\subsection{Data construction and management technologies}

Both the physical entities and the virtual models are driven by data, which are the media by which DT understands, responds and interacts with the real world. The whole lifecycle of DT 
JIMSE 2,1

8

data consists of data generation, data storage, data transmission and data processing. Since data generation mainly refers to perceptions of the outside world, which is discussed in 3.1, and data transmission has a strong association with the connection, which is elaborated in 3.5, this subsection focuses on a survey of data storage and data transmission technologies as follows:

A high-fidelity DT model contains intricate information, such as geometry information, physical information, condition information, etc., where a high volume of data needs to be stored intact. Some IoT technologies, such as bar code, quick response (QR) code and radio frequency identification (RFID), can be utilized to store a certain amount of information. With the development of big data storage frameworks, such as the MySQL database, HBase, NoSQL database, etc., a large amount of data can be correctly arranged and utilized. In MySQL the data are stored in the form of tables, where each row has various record names and each column has concrete values of data. Many rows and columns comprise a form, and several forms compose a database (Ongo and Kusuma, 2018). HBase employs the Hadoop Distributed File System (HDFS) as its file storage system, and Hadoop MapReduce provides HBase with high-performance computing power. Zookeeper provides stable service, and a failover mechanism for HBase (Bhupathiraju and Ravuri, 2014). The feature of NoSQL is to remove the relational feature of relational database. The NoSQL database has very high readwrite performance, especially in large data volume, also excellent performance.

The basic purpose of data processing is to extract and derive data that are valuable and meaningful to particular people from large, potentially cluttered, incomprehensible amounts of data. Raw data are useless unless they undergo data cleaning, compression, smoothing, transformation, reduction, etc. Big data analytics may be divided into the following aspects: analytic visualizations, data mining algorithms and predictive analytic capabilities. Data visualization aims to communicate clearly and effectively with the aid of graphical means. Data can be visualized by different forms of tables and graphics (histograms, bar charts, pie charts, etc.). Data mining generally refers to the process of algorithmically searching for information that is hidden in a large amount of data from a large amount of data. Commonly employed data mining algorithms include K-means algorithms (Kapil et al., 2016), support vector machines, the apriori algorithm, the expectation-maximization algorithm, the nearest neighbor approach (Dröder et al., 2018), the naive Bayesian model and classification and regression trees (CART), etc. Predictive analytics are advanced analytic techniques that leverage historical data to uncover real-time insights and predict future events. These techniques combine a variety of advanced analytics capabilities, including ad hoc statistical analysis, predictive modeling, data mining, text analysis, optimization, real-time scoring, machine learning, etc.

To address multisource heterogeneous data, data fusion is necessary for collection, transmission, synthesis, filtering, correlation and synthesis of useful information from various information sources. There are three levels of data fusion methods: signal-level fusion, feature-level fusion and decision-level fusion (Liu et al., 2018). Data fusion methods include Kalman filtering ( $\mathrm{Li}$ et al., 2017), image regression, principal component transform (PCT), the K-T transform, the wavelet transform, etc. Despite these data fusion methods, few articles discuss the implementation of these concrete algorithms or technologies into DT.

\subsection{Virtual modeling technologies}

As explained in Tao and Zhang (2017) and Tao et al. (2018d), a complete virtual model contains the geometry, physical, behavioral and rule model. Geometry information includes the shape, size, position and assembly relationship. There are diverse mature computer-aided design $(\mathrm{CAD})$ software that can visualize the geometrical information of a physical object, such as UG, AutoCAD, SolidWorks and Creo.

Physical information contains the tolerances (dimensional tolerance, geometrical tolerance, surface roughness, etc.), material properties (density, Young's modulus, 
Poisson's ratio, etc.) and other information. Verner et al. (2018) employed Creo to build a robot DT model, in which the geometry information is recorded and its balance characteristics were calibrated using "center of gravity analysis" and "sensitivity analysis" features of Creo.

A behavioral model describes how the virtual model responds to external stimulates; for example, changes in the outside world, interaction with other objects, etc. Numerous physicsbased theories/models have been established to reveal the mapping relationship between the input and the behavior, such as the computational fluid dynamic (CFD) model, finite element model (FEM) (Tuegel et al., 2011b), robot dynamics model, etc. In shop-floor design, Tao and Zhang (2017) suggest that behavioral models describe the mechanical correspondence of production equipment under the circumstance of a given numerical control program and disturbance, such as human interference, and proposed that descriptions of behavior can be implemented specifically using finite element models and neural networks.

The rule model involves associations and constraints, which can be applied to analyze, optimize and predict the object performance. To extract rule information, several technologies and algorithms can be utilized, such as the data mining algorithm (K-means (Tao and Zhang, 2017) and neural network (Tao et al., 2018d)), semantic data analytics (Abramovici et al., 2016) and XML-based specific data format (AutomationML (Schroeder et al., 2016) and CityGML (Ruohomäki et al., 2018)). Tao et al. (2018d) proposed that in the wind turbine rule model, the limitation of wind speed can be calculated by force analysis and correlations between parameters can be explored via neural networks.

It is important to apply verification, validation and accreditation (VV\&A) technology to evaluate the accuracy of the virtual model (Tao et al., 2018c). Tao and Zhang (2017) applied VV\&A technology to verify the accuracy in the model to the corresponding programming code, simulation confidence, sensitivity and simulation accuracy, etc.

\subsection{Services technologies}

DT services technologies aim to fulfill different objectives in different applications. For example, in aerospace engineering, DT services refer to aircraft structural life prediction (Tuegel et al., 2011b) while in healthcare, DT services focus on monitoring, diagnosing and predicting the health conditions of the elderly (Liu et al., 2019b). The diversity of services leads to the demand for expertise. To generate a service, corresponding data, knowledge and algorithms must be encapsulated where service description and encapsulation technology is needed (Tao and Zhang, 2017; Qi et al., 2018).

A service description refers to an accurate statement of specific demand, e.g. production planning in manufacturing engineering (Tao and Zhang, 2017) and structural monitoring and fault prediction (Tuegel, 2012). Since multiple services are often embedded in a complex system, decomposing services to corresponding subservices and making a smart service selection are important. The real-time visualization of DT services is a key object of DT, which requires computer graphics processing technology, such as computer graphics, 3D rendering and image processing technology.

\subsection{Connection and data transmission technologies}

To realize real-time control and virtual-real state mapping, connection methods with high fidelity are necessary for DT. There are abundant connection protocols for data exchange between the physical space to the DT and inside the cyber space among different software. The existing data transmission method consists of wire transmission and wireless transmission (refer to Figure 3). Wire transmission includes the twisted pair (categories 5 and 6), coaxial cable (coarse and fine) and optical fiber (single-mode and multimode), while wireless transmission includes Zig-Bee, Bluetooth, Wi-fi, ultra-wide band (UWB) and nearfield communication (NFC) (Cheng et al., 2018). For long-distance wireless transmission 
JIMSE 2,1

technology, GPRS/CDMA, digital ratio, spread spectrum microwave communication, wireless bridge and satellite communication are available. An extensive range of application program interfaces (APIs) are commonly employed for data exchange between different software to realize data transmission on the software level. Recently, $5 \mathrm{G}$ technology can be applied to satisfy the demand of high data rates, high reliability, high coverage and low latency (Cheng et al., 2018).

\subsection{Environment coupling technologies}

Similar with DT virtual model, the virtual environment model also contains geometry, physics and behavioral information. Geometric information describes the environment in terms of its geometry and appearance, and presents it in a data format that can be processed by a computer. Physical information contains the mechanical parameters of the environment, which are essential when performing physics-based simulation, e.g. finite element analysis and hydrodynamic analysis. For example, in marine engineering, the mechanical parameters of the ocean, such as density, viscosity, etc., will affect the influence of the marine environment on the operation and performance of marine equipment, such as ships and oil wells. Environmental mapping techniques, sensor acquisition techniques and full element digital definition techniques can be used to describe geometric and physical information about the environment. Environmental mapping technologies include remote sensing technologies, seismic wave method, polarization, radio navigation system, hydroacoustic positioning systems, 3D modeling technology, etc. Behavioral information reflects the change of environment in response to the operation of DT model. For example, during tunneling, the cutting head of the tunnel boring machine (TBM) churn up the rocky soil, which has a significant impact on the subsurface environment. Finite element analysis and computer fluid dynamics can be used to simulate the influence exerted by virtual models in the environment, whereas neural network and surrogate model are also able to be implemented to predict the future state of the environment. The visualization of environment is as prominent as the visualization of virtual model. Due to the large volume of the environment, a multi-channel immersive stereoscopic display method through wearable devices (e.g. head-mounted displays, tactile gloves) combined with virtual reality (VR) and mixed reality (MR) would be suitable for the environment visualization.

\section{DT in categorized applications}

\subsection{DT in product lifecycle phases}

As the complexity of equipment is increasing, the lifecycle cost has increased significantly. PLM is the business activity of managing products across the lifecycle, from the very first idea for a product until it is retired and disposed (Tao et al., 2018a). Traditional computeraided tools (e.g. CAD/CAE/CAM), structural health monitoring, condition and performance monitoring, and other technologies have been applied to the PLM. However, this research focuses on physical products rather than virtual models and lacks the fusion of physical products, virtual models and data. With data and enabling DT technologies, DT can provide services for the entire lifecycle of the product, including design, manufacturing, operation and maintenance, and recycle phases. The potential applications of DT in the four phases of a product lifecycle are investigated as follows: After surveying 32 relevant studies in this section, the proportions of the DT paper that address the four phases are shown in Figure 4.

4.1.1 DT for design. Traditional product design process considers the professional knowledge and experience of the individuals. In this case, the designers must carry out various tests to constantly show the validity and usability of the design at the designing stage 


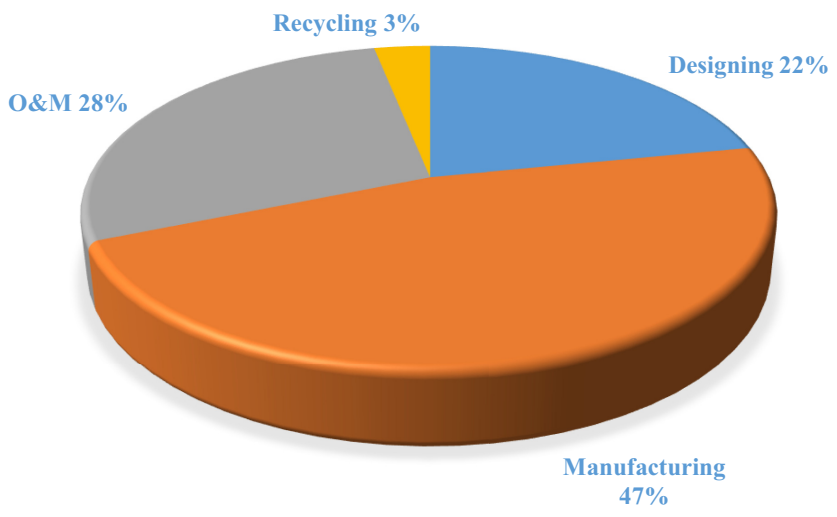

Book review

(Tao et al., 2018a). Furthermore, with the individualized designing demands, more realistic virtual models that mirror the real world of production lines are essential (Zhang et al., 2017). In allusion to these problems, some new product design approaches based on DT have been developed.

Tao et al. (2019) proposed a framework of DTPD, which includes five parts: planning and task clarification, conceptual design, embodiment design, detail design and virtual verification. In the task clarification phase, customer needs are translated into functional requirements with design constraints, where DT serves as an "interpreter" to facilitate the "translation" process. In the conceptual design phase, the functional requirements are mapped to the design parameters, working principles and physical structures. In the virtual verification phase, driven by the incoming physical data, the virtual model is progressively upgraded and optimized. A case of bicycle redesign is presented to illustrate an application of the proposed DTPD method.

To ensure the validity and usability of the DT model for design purposes, key enabling techniques are proposed. For example Zhang et al. (2017), presented a DT-based approach for rapid individualized design of a production line and three key enabling techniques, including reference models, distributed integration and multi-objective optimization between static design and dynamic control. Liu et al. (2019a) proposed four key techniques for DT-driven design of the automated flow-shop manufacturing system (AFMS), including initial, rapid and individualized design based on reference models, real-time cyber-physical synchronization, distributed semi-physical integration, multi-objective optimization of dynamic execution and bi-level programming between static configuration and dynamic execution. Tao et al. (2018a) applied big data technology to DT-based product design. A simulation package from a commercial PLM software has been released to address design challenges in water pumps (Ferguson et al., 2017).

DT technologies have also been implemented in product geometry control in the design phase. For instance, Schleich et al. (2017) propose a comprehensive reference model that is based on the concept of skin model shapes and apply this model to geometrical variations management. Söderberg et al. (2017) propose a DT concept for real-time geometry assurance in design and use a sheet metal assembly example to illustrate this approach. A detailed survey of characteristic features, key technologies and specific applications of the DT in the design phase is provided in Table 3.

4.1.2 DT for manufacturing. Similarly, a DT system for manufacturing has four key components, including physical entity, virtual model, data and service system (Tao and Zhang, 2017). The following three core techniques have been researched: (1) construction of 


\begin{tabular}{|c|c|c|c|c|}
\hline $\begin{array}{l}\text { JIMISE } \\
2,1\end{array}$ & References & Characteristic components & Key technologies & $\begin{array}{l}\text { Specific } \\
\text { application }\end{array}$ \\
\hline \multirow[b]{2}{*}{12} & $\begin{array}{l}\text { Tao et al. } \\
(2019)\end{array}$ & Framework of DTPD & $\begin{array}{l}\text { Planning and task clarification; } \\
\text { Conceptual, embodiment and detail } \\
\text { design; Virtual verification }\end{array}$ & Bicycle redesign \\
\hline & $\begin{array}{l}\text { Schleich et al. } \\
\text { (2017) }\end{array}$ & $\begin{array}{l}\text { Comprehensive reference model } \\
\text { based on skin model shapes }\end{array}$ & $\begin{array}{l}\text { Geometrical variations } \\
\text { management }\end{array}$ & $\begin{array}{l}\text { Geometrical } \\
\text { variations } \\
\text { management }\end{array}$ \\
\hline \multirow{5}{*}{$\begin{array}{l}\text { Table } 3 \text {. } \\
\text { Survey of DT } \\
\text { references for the } \\
\text { design phase }\end{array}$} & $\begin{array}{l}\text { Zhang et al. } \\
(2017)\end{array}$ & $\begin{array}{l}\text { Rapid individualized design of } \\
\text { production line; Platform of } \\
\text { hollow glass production }\end{array}$ & $\begin{array}{l}\text { Distributed integration; } \\
\text { Multiobjective optimization }\end{array}$ & $\begin{array}{l}\text { Hollow glass } \\
\text { production line }\end{array}$ \\
\hline & $\begin{array}{l}\text { Söderberg } \\
\text { et al. }(2017)\end{array}$ & $\begin{array}{l}\text { Real-time geometry assurance; } \\
\text { Design, preproduction and } \\
\text { production phases }\end{array}$ & $\begin{array}{l}\text { Locating scheme optimization; } \\
\text { Statistical variation simulation }\end{array}$ & $\begin{array}{l}\text { Sheet metal } \\
\text { assembly }\end{array}$ \\
\hline & $\begin{array}{l}\text { Liu et al. } \\
\text { (2019a) }\end{array}$ & $\begin{array}{l}\text { Framework of DT-driven design } \\
\text { of AFMS; Rapid individualized } \\
\text { design platform for AFMS }\end{array}$ & $\begin{array}{l}\text { Cyber-physical synchronization } \\
\text { and distributed semiphysical } \\
\text { integration; Multiobjective } \\
\text { optimization of dynamic execution; } \\
\text { Bi-level programming between } \\
\text { static configuration and dynamic } \\
\text { execution }\end{array}$ & $\begin{array}{l}\text { Sheet material } \\
\text { production line }\end{array}$ \\
\hline & $\begin{array}{l}\text { Tao et al. } \\
\text { (2018a) }\end{array}$ & $\begin{array}{l}\text { Big data in product lifecycle; DT- } \\
\text { based product design process }\end{array}$ & $\begin{array}{l}\text { Conceptual design; Detailed design; } \\
\text { Virtual verification; }\end{array}$ & Bicycle design \\
\hline & $\begin{array}{l}\text { Ferguson } \\
\text { et al. (2017) }\end{array}$ & DT platform development & $\begin{array}{l}\text { Using Siemens PLM Software } \\
\text { platform }\end{array}$ & $\begin{array}{l}\text { Water pumps } \\
\text { design }\end{array}$ \\
\hline
\end{tabular}

the DT model; (2) acquisition and management of data and (3) services that consist of prediction and production management (Zhuang et al., 2018).

The DTS concept was proposed in Tao and Zhang (2017), where the four components, i.e. physical shop-floor (PS), virtual shop-floor (VS), shop-floor service system (SSS) and shopfloor DT data (SDTD), its key technologies and current challenges are discussed. The PS consists of manufacturing factors, i.e. human, machines, material and products. The implementation of sensors (e.g. RFID, Zhuang et al., 2018) are needed to collect data and communication interfaces and protocol (such as CoAP, Tao et al., 2017a), which is essential to transmit data and receive commands from the upper level. The VS is considered from the following perspectives: geometry, physics, behavior and rule (Tao and Zhang, 2017; Zhang et al., 2019b; Zhuang et al., 2018; Tao et al., 2017b). SSS encapsulates various data, functions and algorithms to satisfy different demands from the PS and VS, such as production planning and monitoring, models calibration and validation, etc. (Qi et al., 2018; Ciavotta et al., 2017). SDTD collect, converge, store and analyze data generated from the PS, VS and SSS (Tao et al., 2018a). Zhang et al. (2019c) explored the concept and basic components of the PMDT, which consists of five models, i.e. PDM, GSM, MAM, BRM and DFM. Based on the PMDT, a new architecture of the cyber-physical production system (CPPS), which is composed of five layers (i.e. the physical layer, the network layer, the database layer, the model layer, and the application layer), was composed. In Qamsane et al. (2019), the overall DT platform is partitioned into individual DT classes that allow modeling of the major components, such as physical topology, machine assets, machine processes and system processes, and the DT instances of these classes are combined via coordination of the DT manager. Leng et al. (2019) proposed a DT-driven manufacturing cyber-physical system (MCPS) for parallel controlling of a smart workshop, which consists of four parts: the manufacturing workshop, the manufacturing execution, the online parallel controlling and the decentralized selforganizing. 
In the manufacturing process, numerous data will be generated. The collection, fusion and transmission of these data is an important part in constructing DT. The data collected in the physical manufacturing shop-floor are divided into three categories: real-time perception data, production process data and production activity plan data (Zhuang et al., 2018). Multimodal data acquisition has to be conducted. Sensor-based tracking and machine vision are introduced for data acquisition in small and medium-sized enterprises (SMEs) (Uhlemann et al., 2017). The collected data need to be fused and integrated and then uploaded to the database via the network. The $5 \mathrm{G}$ networks contribute higher bandwidth, faster speeds and lower latency. Coronado et al. (2018) developed a mobile Android OS-based manufacturing execution system (MES), and the data obtained from the MES is sent to the cloud and stored in a database, where it is merged with MT Connect data collected from the networked machine tools. The database can use the Hierarchical Data Format Version 5 (HDF5) file format, which provides a flexible structure to enable the storage of multiple different data (Zambal et al., 2018). The data flow of DTs for the manufacturing stage is shown in Figure 5.

With DT model and acquired data, some prediction and production management services will be provided. Zambal et al. (2018) used an analytical model and finite-element model to predict product defects; however, in the case of critical defects, the finite-element model requires a considerable amount of time to generate a result. Regarding the production management and control principles, Leng et al. (2019) proposed the idea of bi-level intelligence between local decentralized self-organizing and holistic online parallel controlling. Malik and Bilberg (2018) explored the human-robot allocation scheme in a production setting and estimate the task completion times. Liau et al. (2017) presented the application of the DT concept in the injection molding industry from mold design to mold making and the injection molding process. In addition, Priggemeyer and Rossmann (2018) explored a technology that allows transparent transfer of the simulation results from a virtual environment to a real system and then apply the virtual system's state to actually control the physical components. The research of DT in the product manufacturing stage is summarized in Table 4.

Globalization contributes new challenges to manufacturers: unpredictable market changes, rapidly varying demands and frequent introduction of new products. In this circumstance, the demand of rapid response to these changes has been proposed. Reconfigurability is a capacity that allows manufacturing systems to add, remove and

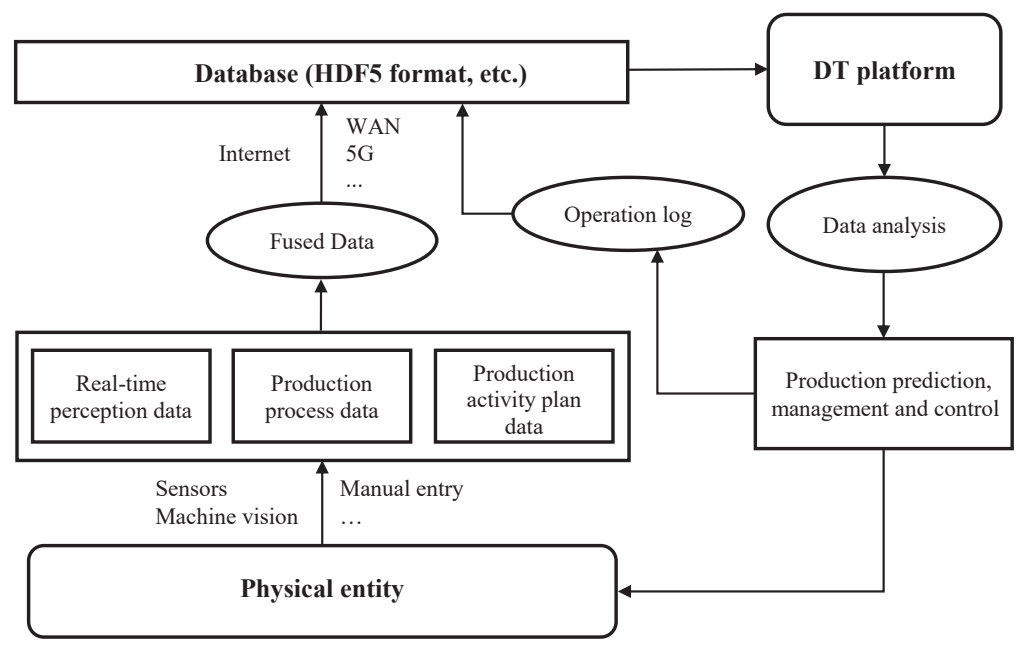

Figure 5. Data flow of DT in manufacturing 


\section{JIMSE 2,1}

\section{4}

Priggemeyer and Simulation-based Control Rossmann (2018)

\section{Zambal et al} (2018)

Malik and Bilberg (2018)

Coronado et al. (2018)

Qamsane et al.

(2019)

Leng et al. (2019)

Zhang et al. (2019c)

Zhuang et al. (2018)

Uhlemann et al. (2017)

Liau et al. (2017)

Stark et al. (2017)

Liu et al. (2019a)

Table 4.

Survey of DT

Malik and references for the manufacturing phase
Characteristic components

PS; VS; SSS; SDTD

PS, VS, SSS, SDTD

Data management and database; Predict mechanical properties

DT framework for HRC workstation

Android OS-based MES; Cloud database

Novel DT architecture: DT pool and DT manager

DT driven manufacturing CPS; Workshop, execution, parallel controlling and decentralized self-organizing

PMDT consists of five models; CPPS composed of five layers

Framework of DT-based smart production; Prediction services for the shop-floor

Composition of a database;

Multimodal data acquisition

DT concept in injection molding industry

Modularized functional design with a construction kit

DT-based design; Execution engine; Production simulation; Multiple optimization; Balancing via bi-level programming

Physical space; Virtual space: simulate production plan, optimize simulation, convert to production plan
Key technologies

Specific application

Perception and access,

Communication protocol analysis,

Data encapsulation and publication; Multidimensional modeling, Model integration, Model verification; Data cleaning, Data integration, Data fusion; Iterative optimization, Selflearning, self-organization, and self-adaption mechanism; Service encapsulation, composition and publication; Demand decomposition; Service-demand matching

Purdue Enterprise Reference Architecture (PERA) and Reference Architectural Model Industry 4.0 (RAMI 4.0)

Structure of database; Finite element calculations

Human-robot task allocation; Human ergonomic analysis Data set integration; Cloud computing and manufacturing DT pool consists of DT classes; DT manager coordinates DT instances Data driven cyber-physical fusion; Triple-view cyber-physical synchronization and integration; Decentralized self-organizing tasks; Holistic online parallel controlling /

Big data technologies

Sensor-based tracking; Machine vision

Internet-of-Things; Cyber Physical System

Virtual prototyping and validation

Initial rapid individualized design of static configuration; Multiview synchronization and distributed semi-physical simulation; Optimal design of dynamic execution; Iterative design between static configuration and dynamic execution

Collision analysis; Reach test; Placement test; Vision test
Shop-floor

Shop-floor design

Robotic Workcell

Aircraft wing

Assembly work station Manufacturing shop-floor Manufacturing flow-shop Smart manufacturing workshop

Aircraft engine blisk machining shopfloor Satellite assembly shop-floor

Small- and mediumsized enterprises Injection molding

Shop-floor reconfiguration Shop-floor reconfiguration reconfiguration 
rearrange components to satisfy the rapid change in the markets. Koren et al. (2018) proposed a reconfigurable manufacturing system (RMS), indicating that DT is an effective way to overcome the complexity of the system, and established the requirement of the efficiency of the developed strategy/algorithm that can be employed in real time. The framework of DT in the shop-floor reconfiguration is shown in Figure 6. Based on the RMS, Zhang et al. (2019b) presented a reconfigured DT (RDT) model based on a five-dimensional RDT model, i.e. geometry, physics, capability, behavior and rule. The reconfiguration strategy is based on a dependency tree. Malik and Bilberg (2018) applied Tecnomatix Process Simulate software to perform human-robot task allocation, workstation layout configuration, human ergonomic

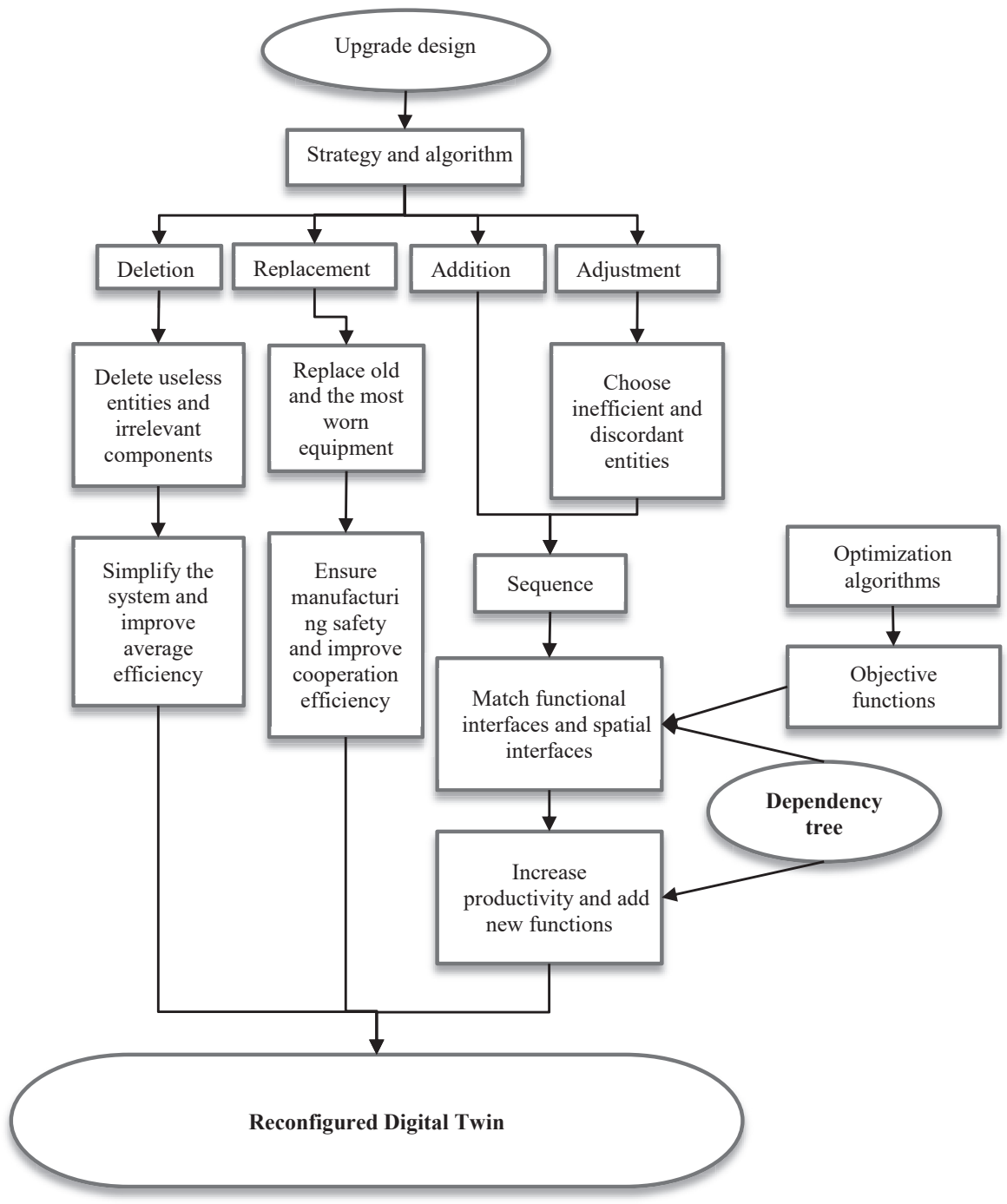

Source(s): Zhang et al. (2019b)

Figure 6. Process of shop-floor reconfigured DT 
JIMSE 2,1

16

analysis in a virtual environment and automatic robot programming. Zhang et al. (2019a) proposed a reconfigurable DT manufacturing system (RDTMS) and reconfigurable strategies for different levels of manufacturing system from the equipment level to the service level. For enterprise applications, DELMIA of Dassault Systems has several roles that can assist in shop-floor design in the virtual environment. There are "shop-floor designer", "shop-floor equipment engineers" and "shop-floor equipment simulation engineer" roles, which aid in the shop-floor layout design and simulation of the production process (Dassult Systems, 2020).

4.1.3 DT for operation and maintenance. Complex equipment, such as aircrafts, ships and wind turbines are designed to work throughout decades in harsh environments. Thus, performance degradation is inevitable during the operation of this equipment, which leads to malfunctioning and high maintenance costs. Therefore, developing a DT for complex equipment is essential to monitor the equipment condition, perform diagnoses and prognoses, and provide design rules for maintenance (Tao et al., 2018d).

Tao et al. (2018d) developed a five-dimensional DT model and presented the framework and workflow of the DT-driven PHM for wind turbines. In this architecture, DT is modeled in five dimensions: physical entity (PE), virtual equipment (VE), services for PE and VE (Ss), DT data (DD), and connection among PE, VE, Ss and DD (CN). In the study by Magargle et al. (2017), to realize the simulation-based DTs, several methods of systems, circuits and reduced order modeling were shown using 3D finite element analysis and OD multidomain circuit simulation. As the physical device runs, its state will change, so we need to update its DT model. Aivaliotis et al. (2019) propose a three-phase modeling scheme, which consists of machine modeling, virtual sensors modeling and model parameters updating. In Wang et al. (2019), a parametric sensitivity analysis-based model updating scheme is investigated to enhance the model adaptability, and particle swarm optimization (PSO) is employed as an optimization algorithm for model parameters updating.

In addition to virtual models, data serve as the key for the DT used in operation and maintenance (O\&M). In Boschert et al. (2018), the concept of products fleet data is proposed and analyzed; a similarity search using a knowledge graph that comprises all devices of a fleet provides knowledge for a specific device by searching for similar devices in a fleet. However, there are challenges in the O\&M of fleet equipment: (1) the large amount of collected data that need to be processed and (2) the variability between equipment of the fleet. By using an adaptive physics-based model as a DT for fault detection, isolation and identification, the challenges of considerable variations within equipment in the fleet can be addressed (Zaccaria et al., 2018). In some cases, enough real-time data are not available for simulation models. Monte Carlo simulation can be performed to generate fleet data (Zaccaria et al., 2018). Both the signature-based technique and the machine learning approach can be utilized for data processing; the machine learning approach includes the random forest regressor (RFR), support vector regressor (SVR), gradient boosting regressor (GBR) and artificial neural network (ANN) (Zaccaria et al., 2018; Balakrishnan, 2019).

With the DT model and data, some applications of DT in the product O\&M phase are developed. Tao et al. (2018d) applied DT technology to the prognostics and health management (PHM) of wind turbines. Sivalingam et al. (2018) proposed a physics-based methodology to accurately predict the damage accumulation and remaining useful life (RUL) for an offshore wind turbine power converter by accounting medium- and short-term thermal transient loadings and long-term thermal loading. Li et al. (2017) applied the concept of a dynamic Bayesian network (DBN) to build a versatile probabilistic model for the diagnosis and prognosis of the aircraft wing fatigue crack growth. A particle filter is utilized as the Bayesian inference algorithm for the nonlinear and non-Gaussian DBN. This paper also showed that the DBN can be modified with reasonable assumptions about the measurement error and load observation, which provides substantial computational savings. The research 
of DT in the product O\&M stage is summarized in Table 5. According to these literatures, the framework of the DTs for the O\&M stage is summarized in Figure 7.

4.1.4 DT for recycling. Recently, DT technologies have been implemented in recycling, recovery and remanufacturing. For example, a DT-based system has been developed for WEEE recovery and international standard-compliant data models to support the manufacturing/remanufacturing operations throughout a product's lifecycle, from design to recovery. Users can update the product status via various Industry 4.0 supports, e.g. mobile apps. When an electrical and electronic equipment (EEE) device stops service, users can update the digital status. Recyclers can start the recycling process and determine the recycling mode, material recycling or component recycling based on cloud data recorded in a DT-based system (Wang and Wang, 2019).

\subsection{DT in different engineering fields}

In this section, 26 papers are reviewed to categorize DT applications in different engineering fields. The distribution of these papers in four industrial applications is shown in Figure 8. The remainder of this section provides a brief introduction about how DT is implemented in different engineering fields. Corresponding frameworks are discussed, and the relevant models and technologies are demonstrated.

4.2.1 DT for aerospace engineering. The conventional design features include the usage of a factor of safety and reference to previous design, which hinders the maximum performance of a structure and material. Future aircrafts need to work in more complex environments while reducing their weight (Glaessgen and Stargel, 2012). DT was technically applied in the aerospace engineering area due to the contribution of NASA and US Airforce Research Laboratory. The purpose of DT in aerospace engineering is to reduce the weight of aircraft, even under more complex working conditions, and to mirror the life of the flying twin, which enable unprecedented safety and reliance (Glaessgen and Stargel, 2012; Tuegel et al., 2011a).

\begin{tabular}{|c|c|c|c|}
\hline References & Characteristic components & Key technologies & Specific application \\
\hline $\begin{array}{l}\text { Sivalingam et al. } \\
\text { (2018) }\end{array}$ & $\begin{array}{l}\text { Framework for DT platform; } \\
\text { Damage accumulation and } \\
\text { RUL prediction }\end{array}$ & $\begin{array}{l}\text { Virtual sensors; Monte Carlo } \\
\text { Markov Chain (MCMC) }\end{array}$ & $\begin{array}{l}\text { Offshore wind } \\
\text { turbine power } \\
\text { converter }\end{array}$ \\
\hline $\begin{array}{l}\text { Magargle et al. } \\
(2017)\end{array}$ & $\begin{array}{l}\text { Simulation-based DT model; } \\
\text { Heat monitoring and } \\
\text { predictive maintenance }\end{array}$ & $\begin{array}{l}\text { 3D finite element analysis; 0D } \\
\text { multidomain circuit simulation; } \\
\text { Difference analysis }\end{array}$ & $\begin{array}{l}\text { Automotive } \\
\text { braking system }\end{array}$ \\
\hline $\begin{array}{l}\text { Wang et al. } \\
\text { (2019) }\end{array}$ & $\begin{array}{l}\text { Parameter sensitivity } \\
\text { analysis-based model } \\
\text { updating scheme }\end{array}$ & Particle swarm optimization & $\begin{array}{l}\text { Rotating } \\
\text { machinery }\end{array}$ \\
\hline $\begin{array}{l}\text { Aivaliotis et al. } \\
\text { (2019) }\end{array}$ & $\begin{array}{l}\text { Physics-based DT modeling; } \\
\text { Three phases of modeling }\end{array}$ & $\begin{array}{l}\text { Machine modeling; Modeling of } \\
\text { virtual sensors; Updatable } \\
\text { modeling parameters }\end{array}$ & Industrial robot \\
\hline $\begin{array}{l}\text { Zaccaria et al. } \\
(2018)\end{array}$ & $\begin{array}{l}\text { Fleet data in DT model; } \\
\text { Adaptive physics-based } \\
\text { model }\end{array}$ & $\begin{array}{l}\text { Monte Carlo simulation; Signature- } \\
\text { based technique; NN model }\end{array}$ & Aircraft engines \\
\hline Li et al. (2017) & $\begin{array}{l}\text { DBN for model building; DT } \\
\text { vision for diagnosis and } \\
\text { prognosis }\end{array}$ & $\begin{array}{l}\text { Particle filter for nonlinear and } \\
\text { non-Gaussian DBN; Modify DBN } \\
\text { structure with reasonable } \\
\text { assumptions }\end{array}$ & Aircraft wing \\
\hline $\begin{array}{l}\text { Balakrishnan } \\
\text { et al. }(2019)\end{array}$ & $\begin{array}{l}\text { Develop DT for puppet car; } \\
\text { Dataset transmission and } \\
\text { analysis }\end{array}$ & $\begin{array}{l}\text { Open-source software platform; } \\
\text { Transmit dataset via Hono to Kura; } \\
\text { RVR, SVR and GBR }\end{array}$ & $\begin{array}{l}\text { Automobiles } \\
\text { engine }\end{array}$ \\
\hline
\end{tabular}




\section{JIMSE 2,1}

\section{8}

Figure 7.

Framework of DT for O\&M

\section{Figure 8.}

Distribution of papers about DT in various engineering fields
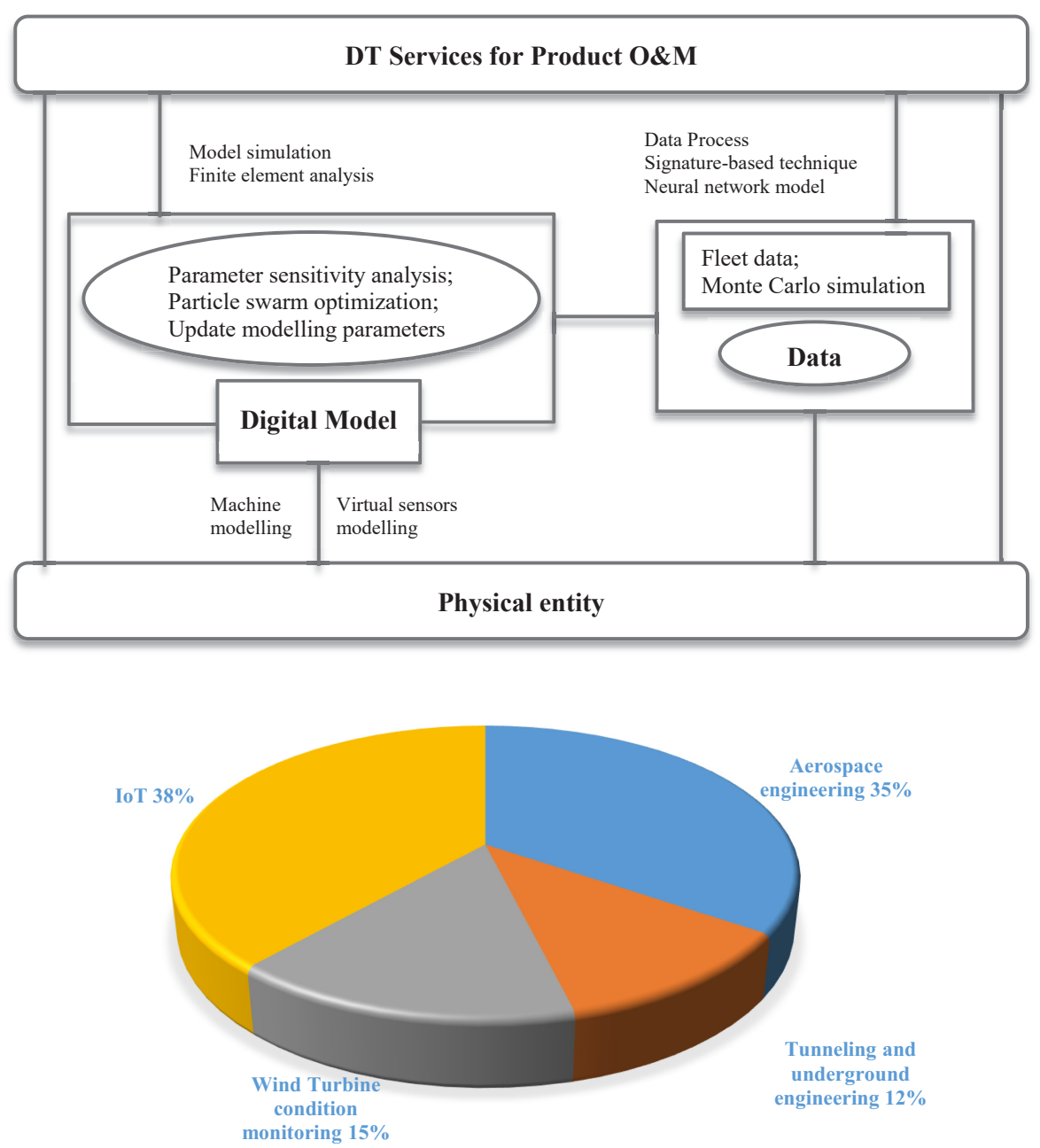

One of the DT frameworks in aerospace engineering is named the Airframe DT (ADT), which is aimed at the operation and maintenance $(\mathrm{OM})$ stage. As shown in Figure 9, by receiving the fleet data according to diverse missions and trajectories, which contains information about motion of the vehicle (Gockel et al., 2012), a CFD model calculates the aerodynamic loads applied to the airplane. These loads are subsequently transmitted to an FEM that computes the response of the aircraft, such as the vibration and stress/strain responses. The resulting damage is simulated and accumulated for RUL evaluation (Tuegel et al., 2011b). Gockel et al. (2012) took advantage of the ADT in a touch-and-go practice and measured the response with discrete time.

To sense the change in the external environment and validate the DT model, some sensitive technologies have been developed. Hochhalter et al. (2014) put an Ni-Ti sensory particle into a specimen using standard manufacturing skills, which can sense microcracks and replicate the geometry of the specimen with support of X-ray CT. Reifsnider and Majumdar (2013) presented a multidisciplinary physics-based methodology to predict the life 


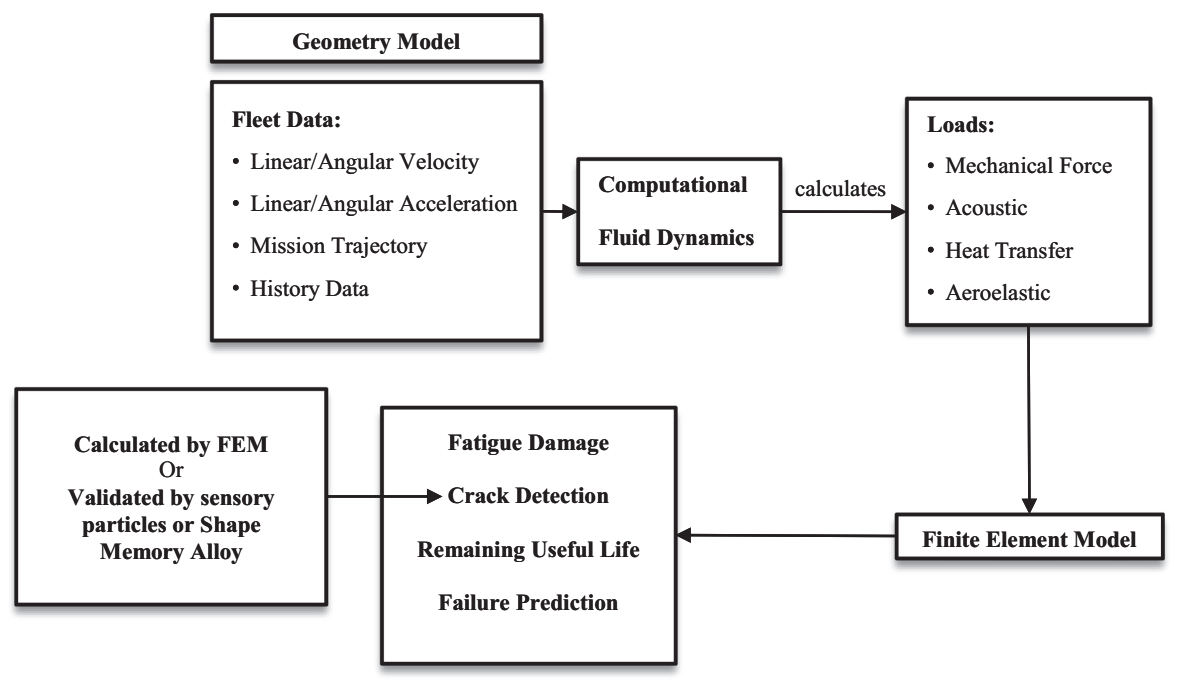

Book review

of a flying DT and utilized dielectric spectroscopy to indicate material emergent behavior. Bielefeldt et al. (2015) developed a nondestructive evolution (NDE) method for an aircraft wing, whose key region was embedded with shape memory alloy (SMA) as sensor to detect crack initiation and development. Inversely, the virtual model can be employed as a virtual sensor to simultaneously monitor the condition of an airplane and reduce the frequency and cost of repair and maintenance (Tuegel, 2012). The DT references and ADT component are summarized in Table 6. We can imply that CFD and FEM are concurrently implemented in an $\mathrm{ADT}$ framework, whereas sensing technology is researched independently.

Another framework is referred to as the industrial digital mock-up (iDMU), which covers the beginning of life (BOL) and middle of life (MOL). The iDMU supports different product structures, addresses the issues related to the integration of functional and industrial design, and facilitates the creation of a collaborative deliverable to support the manufacturing, sustaining and servicing of the aircraft (Ríos et al., 2015). This framework is already successfully applied by Airbus in its A400M final assembly line (FAL) (Mas et al., 2013, 2014). Based on this framework, Ríos et al. (2016) applied Dassault Systemès V6 solutions for data interoperability, manufacturing and assembly, as well as testing and maintenance, using incidences of the aircraft and enabling the biunivocal relation between the physical counterpart and the digital counterpart.

4.2.2 DT for tunneling and underground engineering. Underground engineering equipment is an important complex high-end equipment for infrastructure construction in the national railway, urban construction, water conservancy, mining and national defense fields. Its service environment often has high uncertainty, high ground stress, high water pressure and other characteristics, difficult to characterize with a simple mathematical physical model. The complex structure of underground engineering equipment, the operation process involves machinery and soil interaction simulation requires substandard high computational costs. As an emerging technology that straddles the gap between the physical and virtual worlds of complex products, DT provides a new way to solve the problems of design, manufacturing, operation and maintenance of underground engineering equipment to meet the requirements of complex underground conditions through simulation, monitoring, diagnosis and prediction, optimization and control. 
JIMSE 2,1

\begin{tabular}{llll}
\hline References & Characteristic components & Key technologies & $\begin{array}{l}\text { Specific } \\
\text { application }\end{array}$ \\
\hline Tuegel et al. (2011a) & $\begin{array}{l}\text { Thermal/heat transfer model; } \\
\text { Dynamics model; Stress } \\
\text { analysis model; Fatigue model }\end{array}$ & $\begin{array}{l}\text { Multiphysics modeling, } \\
\text { multiscale damage modeling, } \\
\text { integration of structural FEM } \\
\text { and damage models; Uncertainty }\end{array}$ & $\begin{array}{l}\text { Aircraft } \\
\text { structural life } \\
\text { prediction }\end{array}$ \\
& & and
\end{tabular}

\section{0}

Glaessgen and

Stargel (2012)

Gockel et al. (2012)

Reifsnider and

Majumdar (2013)

Hochhalter et al.

(2014)

Bielefeldt et al.

(2015)

Ríos et al. (2016)

Seshadri and

Krishnamurthy

(2017)

Table 6.

Li et al. (2017)

DT references in aerospace engineering
Integrated vehicle health management (IVHM) system; Ultra-high-fidelity physical models; Sensor updates; Historical norms

Flight profile, CFD; FEM

Interpolation of the principle of microdefect initiation, accumulation, interaction and coalescence in composite materials

Acoustic emission (AE) for tensile test; Combination of geometry and over-the-counter software to improve prediction NDE method for crack detection; Computational demonstration by FEM of aircraft wing; High sensitivity

Based on a commercial software system to accelerate the creation of aircraft digital counterpart

Damage characterization using a wave propagation method at multiple sensor locations; Using Dynamic Bayesian Network for diagnosis and prognosis quantification, modeling, and control; Manipulation of large, shared databases; Highresolution structural analysis capability

Sensor materials and highfidelity response simulation; Design and certification methods; Mission loads and environmental monitoring; Life prediction and extension, etc. Reasonable computational effort; Discretization of flight profile; Data storage; Interpolation of loads and stress/strain results; Mapping; Analysis of discretized points

Dielectric spectroscopy for detecting micro defects

AE sensing technology; Digital image correlation (DIC)

Improve safety and reliability

Generate history of flight loads

Shape memory alloys (SMA) embedding

Computer-aided tools (CAX); Product lifecycle management (PLM); Enterprise resource planning (ERP); MES

Wave propagation analysis

Particle filter; Degeneracy, sample impoverishment, and resampling
Crack detection

Prognosis of composite structure

Crack detection

Aircraft industrial design

Accurate damage detection Diagnosis and prognosis

Many tunneling and underground engineering companies have paid much attention to research and application of DT. DigitalTwin Technology in German has raised a solution called Tunnelware, where the working status of underground engineering equipment can be diagnosed by tunnel designer, owner and the technical staff together (Tunnelware, 2020). Germany Bauhaus Universität established a general tunnel information modeling framework, proposed a numerical simulation-based ground settlement prediction method and developed an interactive display system for ground settlement timing process caused by tunnel construction (Beer, 2003). Kim and Kim (2020) used DT technology for noise barrier tunnels' 
(NBTs) lifespan prediction where gyro sensors were implemented to analyze the change of physical component's behavior and a simple numerical method was utilized for fast lifespan prediction. The Neanex BIM portal contains a "twin" representation of the real physical tunnel, which can be navigated in 3D and displays all the necessary information for the highlighted individual asset (Neanex, 2020). The software uses graphics technology (NoSQL) to process large amounts of data, is completely based on cloud computing, and provides ready-to-use additional components for Autodesk (CAD) applications, system engineering tools, and the related data platform Laces, for sharing across supply chains under open standards data. Research on the DT in underground engineering equipment to date is summarized in Table 7 including their characteristic and key technologies, where year information may be missing as some of the research results come from company websites.

There are plenty of researches about the mechanism of how underground equipment influences the environment. Liao et al. (2009) summarized and elaborated the ground movement prediction based on field measurements and site investigations of actual projects in Shanghai soft ground. Chen et al. (1999) estimated free-soil movement based on analytical method and imposed these movements on the pile to compute the pile response. Finno and Clough (1985) used FEM and field observation to gain an understanding of ground behavior around Earth pressure balance (EPB) shields. However, these mechanisms and models have not been considered during the construction of underground DT, leading to the incompleteness of DT model and unguaranteed DT simulation confidence level. Thus, problems such as the simulation-based optimization design coupled with complex service environment and precise prediction and matching of the state of construction operations with the geological environment during O\&M phase still remains to be solved. The construction of environmental in digital form, the mapping between geological environment and equipment operational status and AI-based technology for geo-environmental prediction and fault prediction need to be researched.

4.2.3 DT for wind engineering. As complicated equipment, a wind turbine (WT) is designed to work in a complex environment for a long time. Thus, the PHM has an important role. By implementing DT, a real-time model of the equipment is visualized, where the usage condition, such as wear, cracks and remaining service life, etc., can be predicted (Gao et al., 2015). Tao et al. (2018d) established a seven-step DT approach to implement DT in PHM (as shown in Figure 10) and employed it on the WT as a case study. SAP's DT for wind power monitoring (Erikstad, 2017) enables operators to implement adaptive control strategies and improved predictive maintenance tactics based on the physical condition of the system at any time, using a digital representation of the real asset. This DT solution supports maintenance operations and structural capability utilization. Bazilevs et al. (2015) developed a dynamic data-driven application system (DDDAS) for fatigue-damage modeling in large-scale laminated composite materials and applied it in the full-scale fatigue test of CX-100 blade.

\begin{tabular}{|c|c|c|c|c|}
\hline References & Characteristic & Key technologies & Specific applications & \\
\hline $\begin{array}{l}\text { Tunnelware } \\
(2020)\end{array}$ & $\begin{array}{l}\text { Intelligent Connection, } \\
\text { Segment management }\end{array}$ & $\begin{array}{l}\text { Building Information Model (BIM), } \\
\text { Cloud solution, Data analytics and } \\
\text { visualization, Sensor data } \\
\text { management }\end{array}$ & $\begin{array}{l}\text { Tunnel construction } \\
\text { operation and } \\
\text { management }\end{array}$ & \\
\hline $\begin{array}{l}\text { Kim and Kim } \\
(2020)\end{array}$ & $\begin{array}{l}\text { Basis research to combine } \\
\text { DT with noise barrier } \\
\text { tunnel (NBT) }\end{array}$ & $\begin{array}{l}\text { Sensor data process, data } \\
\text { correlation, numerical model } \\
\text { analysis }\end{array}$ & NBT life analysis & $\begin{array}{r}\text { Table } 7 . \\
\text { DT references in }\end{array}$ \\
\hline Neanex (2020) & $\begin{array}{l}\text { Integrates requirements, } \\
\text { design and asset } \\
\text { management information }\end{array}$ & $\begin{array}{l}\text { Graphics technology, Cloud } \\
\text { computing }\end{array}$ & $\begin{array}{l}\text { Tunnel virtual model } \\
\text { construction }\end{array}$ & $\begin{array}{r}\text { tunneling and } \\
\text { underground } \\
\text { engineering }\end{array}$ \\
\hline
\end{tabular}




\section{JIMSE 2,1}

\section{2}

Figure 10.

Framework of DT in wind turbine condition monitoring

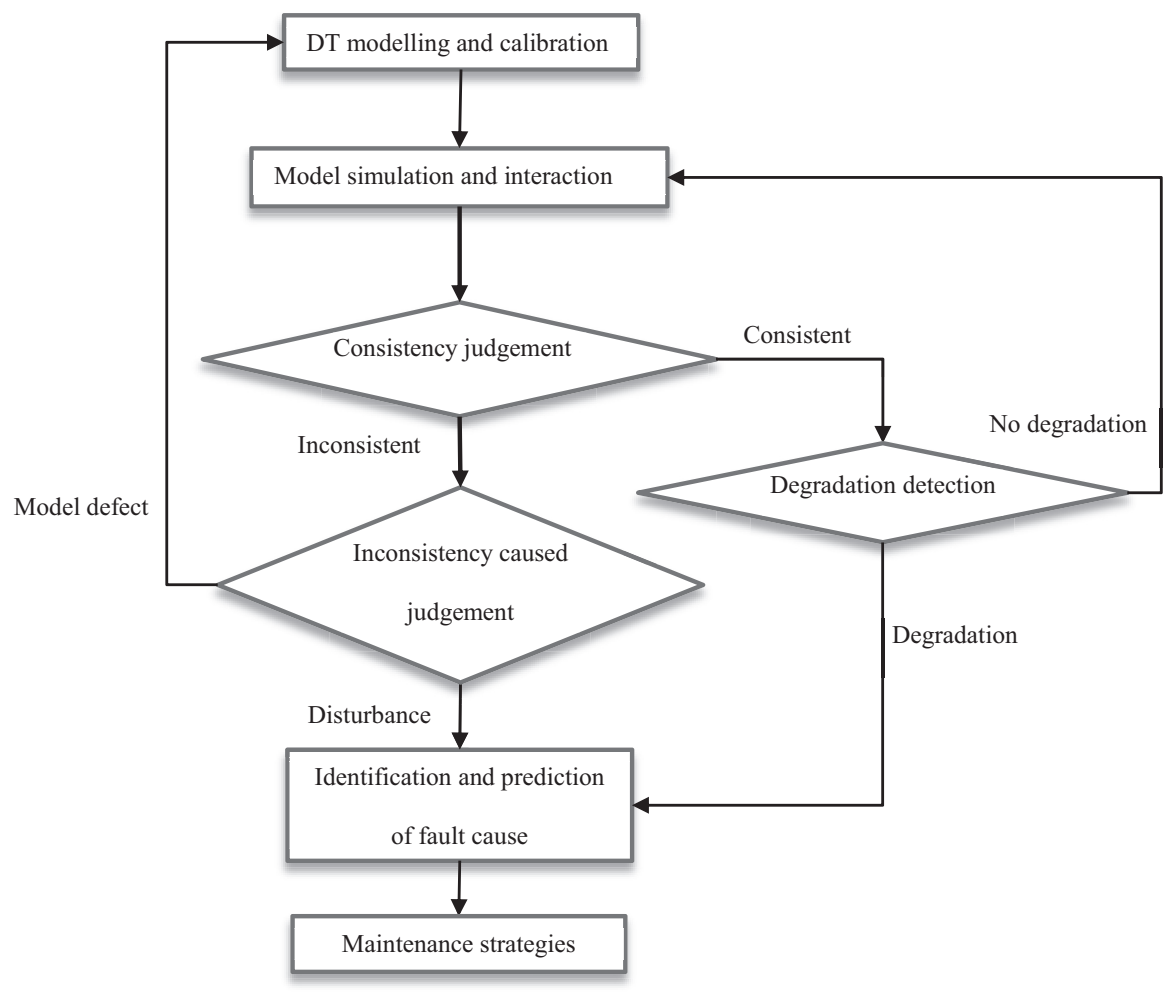

Source(s): Tao et al. (2018d)

Numerous DT platforms and wind power companies, such as GE (Ge, 2020a, b) and DNV GL (Ghobadi, 2017), employed DT models to monitor WTs in wind farms. These models are supplied with real data from sensors and can serve as virtual sensors to simulate different scenarios, such as observing the influences of changes in the wind flow. A 5MW NREL numerical turbine (Sivalingam et al., 2018) was developed for power converter RUL prediction under medium- or short-term thermal cyclic loads. Combining the IGBT and diode junction temperature prediction for the next $5 \mathrm{~h}$, the RUL estimation algorithm will demonstrate the damage accumulation based on the rain flow counting algorithm, which can be employed for quick and optimal decision-making. The DT frameworks related to WT condition monitoring is shown in Table 8, including its components and key technologies.

In terms of environmental coupling, high-relative-speed objects such as raindrop, atmosphere particles and sand, wind speed and other environment factors need to be considered. Hu et al. (2021) proposed a new smoothed particle hydrodynamic approach which described the erosion effect of raindrops on WT blades from the perspective of the raindrop size, impact speed, impact angle and raindrop shape on the impact stress. Fiore and Selig (2014) presented a numerical method to analyze the impact of collision of insects and sand grains where a two-dimensional inviscid flow field solver coupled with a particle position predictor code was used. Noda and Flay (1999) described a simulation model to examine the effect of mean wind speed, wind shear and vertical wind component. Also, the weather change in response to the operation of wind turbine may be taken into consider. 


\begin{tabular}{|c|c|c|c|c|}
\hline References & Characteristic components & Key technologies & Specific applications & \\
\hline $\begin{array}{l}\text { Bazilevs et al. } \\
(2015)\end{array}$ & $\begin{array}{l}\text { Wind turbine blade and its iso- } \\
\text { geometric analysis (IGA) model; } \\
\text { Sensors; Test data }\end{array}$ & $\begin{array}{l}\text { Iso-geometric analysis (IGA); } \\
\text { Structural Health } \\
\text { Management (SHM); Data- } \\
\text { driven simulation }\end{array}$ & Damage prediction & \\
\hline $\begin{array}{l}\text { Erikstad } \\
(2017)\end{array}$ & $\begin{array}{l}\text { Overview of operations; Wind } \\
\text { farm outline; Real-time } \\
\text { visualization of specific wind } \\
\text { turbine; Asset Component } \\
\text { Analytics \& Model-Based User } \\
\text { Experience }\end{array}$ & $\begin{array}{l}\text { Sensors; Data analytics; } \\
\text { Machine learning; AI }\end{array}$ & $\begin{array}{l}\text { Comprehensive } \\
\text { management of WT } \\
\text { farm }\end{array}$ & 23 \\
\hline $\begin{array}{l}\text { Sivalingam } \\
\text { et al. (2018) }\end{array}$ & $\begin{array}{l}\text { Risk assessment; Revenue loss } \\
\text { calculator; safe weather operation } \\
\text { window prediction; maintenance } \\
\text { records; Digital turbine with } \\
\text { SCADA integration with AI; } \\
\text { Physics-based models for RUL } \\
\text { prediction }\end{array}$ & $\begin{array}{l}\text { Virtual sensors; Fast } \\
\text { modeling; Machine learning/ } \\
\text { AI }\end{array}$ & $\begin{array}{l}\text { Remaining Useful } \\
\text { Life prediction }\end{array}$ & \\
\hline $\begin{array}{l}\text { Tao et al. } \\
\text { (2018d) }\end{array}$ & $\begin{array}{l}\text { Physical entity (PE); Virtual } \\
\text { equipment model (VE); Services } \\
\text { model (Ss); DT data model (DD); } \\
\text { Connection model (CN) }\end{array}$ & $\begin{array}{l}\text { Sensor; Modeling; Data } \\
\text { process; Connection }\end{array}$ & $\begin{array}{l}\text { Fault cause } \\
\text { prediction }\end{array}$ & $\begin{array}{r}\text { Table } 8 . \\
\text { DT platforms for WT } \\
\text { condition monitoring }\end{array}$ \\
\hline
\end{tabular}

Barrie and Kirk-Davidoff (2010) surveyed the impact of wind turbine installation on atmosphere circulation, which would in turn influence the fatigue load of wind turbine blade.

4.2.4 DT for IoT applications. The IoT is a system of interrelated computing devices, mechanical and digital machines, objects, animals or people that have unique identifiers (UIDs) and the ability to transfer data over a network without requiring human-to-human or human-to-computer interaction (Wikipedia, 2020). The IoT adds data that can be employed in manufacturing, health care, automotive, urban management and other industries. DT is the ability to take a virtual representation of the elements and the dynamics of how an IoT device operates and lives throughout its lifecycle. By implementing DT, seamless integration between IoT and data analytics can be solved (Fuller et al., 2019) and visualized. A virtual environment is created for a developer to monitor the condition of devices or test new solutions without building a real physical environment. In this section, the use of DT for supporting IoT applications in smart cities, healthcare and manufacturing is discussed. Table 9 categorizes applications of DT in the IoT in different industries.

The DT city concept model is shown in Figure 11. A variety of sensors are distributed in every corner of the city. For example, mobile phones provide the locations of individuals and cameras upload image information of street conditions. Data are transmitted to the IoT platform to perform different smart city services and then are transmitted to a virtual city to keep pace with the real city (Mohammadi and Taylor, 2017; Ruohomäki et al., 2018; Castelli et al., 2019).

Regarding DT in healthcare, several studies address healthcare platforms or the healthcare DT model. Saddik (2018) compared DT with the human body and proposed that DT could be applied for illness prediction, well-being improvement and lifestyle decisions. Liu et al. (2019b) proposed a cloud DT healthcare framework (CloudDTH) for real-time supervision and accurate alerting of emergencies for the elderly in healthcare services.

Currently, most research on DT and IoT focuses on the manufacturing industry. With the development of big data, cloud computing, machine learning and AI technology, a massive amount of data generated during production can be processed, and the industrial IoT (IIoT) advents specifically for the industrial field. 


\begin{tabular}{|c|c|}
\hline References & Characteristic components \\
\hline $\begin{array}{l}\text { Mohammadi } \\
\text { and Taylor } \\
\text { (2017) }\end{array}$ & $\begin{array}{l}\text { Real city; DT city; Spatiotempora } \\
\text { flux; VR }\end{array}$ \\
\hline $\begin{array}{l}\text { Ruohomäki et al. } \\
\text { (2018) }\end{array}$ & $\begin{array}{l}\text { Open standards; Helsinki } \\
\text { 3D + city model; DT; Energy and } \\
\text { climate Atlas; City as a platform; } \\
\text { IoT on urban platform; General } \\
\text { Data Protection Regulation } \\
\text { (GDPR) and privacy }\end{array}$ \\
\hline $\begin{array}{l}\text { Castelli et al. } \\
\text { (2019) }\end{array}$ & $\begin{array}{l}\text { City physical system; Data } \\
\text { collection; Data lake; City } \\
\text { knowledge and models; modeling } \\
\text { and simulation; City DT; City } \\
\text { governance }\end{array}$ \\
\hline Saddik (2018) & $\begin{array}{l}\text { Representation; Intelligence; } \\
\text { Sensing/actuating; Tactile Interne }\end{array}$ \\
\hline
\end{tabular}

Liu et al. (2019b) Physical object; Virtual object; Health care DT data; Cloud health care service platform

Rosen et al.

(2015)

Schroeder et al. (2016)

Alam and

Saddik (2017)

Cheng et al. (2018)

Chhetri et al. (2019)
Part ID and type; Production order number and priority; Production workflow; Current states and locations; $\mathrm{NC}$ program files; Production history Physical components; AutomationML; Middleware; External systems Physical things; Cyber thing; hierarchy-based composition of subsystems; Relationship network; Integration of web services

Enhance mobile broadband (eMBB), massive machine type communication (mMTC) and ultrareliable and low latency communication (URLLC)

DT product parsing; Feature extraction; Synchronize and segment; Clustering algorithm; Anomaly localization algorithm
Table 9.

DT references in IoT
Specific application

Smart City

Modeling; Semantic data management

IoT networks; Data science and modeling; High performance computing; Advanced numerical optimization

Augmented, virtual and MR;

Health care

Haptics; Robotics; $5 \mathrm{G}$ and tactile Internet; Cloud computing;

Wearables; IoT; AI

Health care resource access;

Health care information fusion and sharing; Health care service management; Model management; Health care data security; Data management and analysis /

Data exchange

Cloud computation

VS modeling; Simulation and verification; Health management; Product quality analysis; Shop-floor energy consumption optimization and prediction; Material cracking and rationing; Collaborative production process analysis Side-channel sensing; Data clustering; Anomaly location 


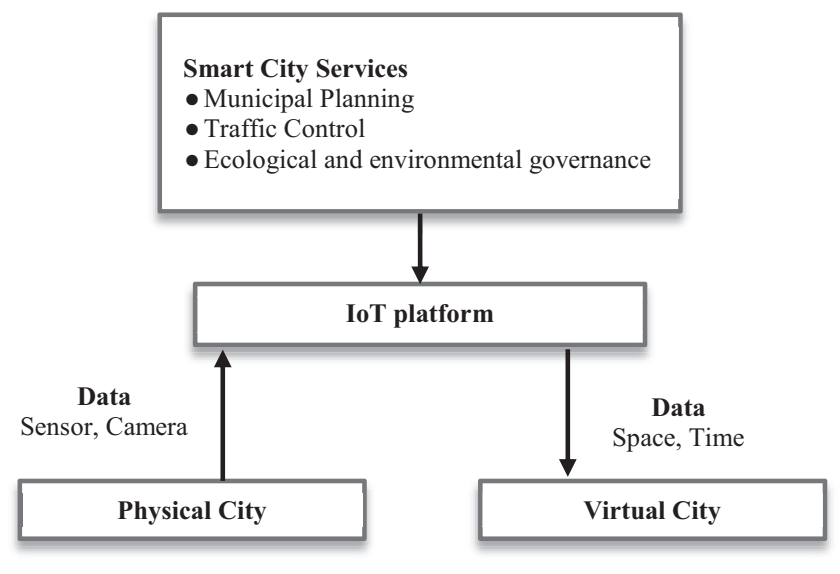

Book review

The DT framework employed in the IIoT is shown in Figure 12. The IoT has provided various sensors for manufacturing equipment, such as a CNC lathe to percept the environment. Additionally, Chhetri et al. (2019) utilized side channels (such as acoustic emission and magnetic) for legacy manufacturing systems without built-in sensors to reveal the cyberphysical relationship of the system. With the implementation of 5G technology, enhance mobile broadband (eMBB), massive machine type communication (mMTC) and ultrareliable and low latency communication (URLLC) can achieve high data rates, high reliability, high coverage and low latency data transmission (Cheng et al., 2018). Schroeder et al. (2016) proposed AutomationML as a data exchange format for different systems. For the IIoT services, cloud-based computing is mentioned in Alam and Saddik (2017) and Zheng et al. (2018) to ensure the scalability of storage, computation and cross-domain communication capabilities.

\section{Key challenges in constructing DT}

Despite the relatively comprehensive five-dimensional DT model with its existing key technologies and previously mentioned applications of DT, some technical issues (e.g. computational effort and data transmission rate) still hinder the development of DT. The

main challenges in constructing DT can be summarized as the following five aspects:

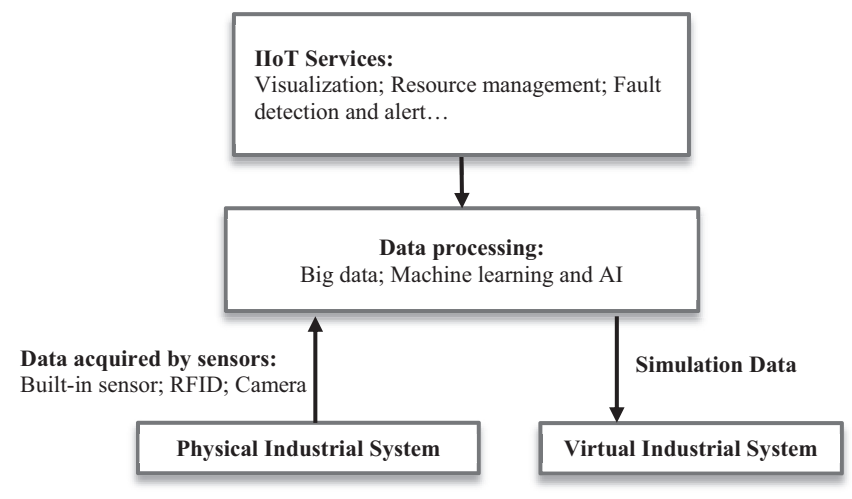

Figure 12. DT in IIoT 
JIMSE 2,1

(1) High-fidelity modeling. Due to the variability, uncertainty and fuzziness of physical space, building models in virtual space to mirror entities with high fidelity is a fundamental issue. Virtual models ought to be faithful replicas of physical entities, which reproduce physical geometries, properties, behaviors and rules (Tao et al., 2019). Current modeling is usually limited to geometric consistency, and much work is needed at the other three levels. With the operation of equipment, the physical entity will change or degrade to a certain extent, and the built model will be inconsistent with the entity. When inconsistences between models and entities appear, how to appropriately identify and update them is difficult. Current modeling generally focuses on a specific life stage of a product. Building a virtual model of a product throughout its entire life-cycle, including design, manufacturing, operation and maintenance, and recycling, is valuable but challenging.

(2) Data acquisition and processing. Data comprise another key driver of DT, which consists of multitemporal scale, multidimension, multisource and heterogeneous data. The whole data lifecycle includes data collection, transmission, storage, processing, fusion and visualization (Tao et al., 2018b). To solve these problems, we need to integrate sensors, machine vision, Internet, IoT, database, data fusion and other technologies. In the previous sections, some data processing approaches, such as signature-based techniques, various neural networks, etc., were discussed. However, some methods have low accuracy, are time-consuming, and consume an excessive amount of computing resources. To ensure real-time and reliable simulation analysis results, we need to develop some fast data analysis methods with high accuracy. In addition, we have to address the problems of fleet data, considering both common characteristics and individual differences of batch products.

(3) Real-time, two-way connection between the virtual space and the real space. The virtual model obtains real-time data of physical entities, and the analysis results are utilized to guide the physical entities in real time. Due to the large amount of data, network transmission delays, model analysis time, etc., it is difficult for DT to achieve a real-time, two-way connection. We also need to solve problems such as visualization and human-equipment interaction.

(4) Unified development platform and tools. Due to different formats, protocols and standards, current tools may not be simultaneously integrated and applied for a particular objective (Tao et al., 2018b). Therefore, the development of a universal design and development platforms and tools for DT are required in the future.

(5) Environmental coupling technologies. The current DT lacks association with external environment. The mechanism explaining how physical object interacts with environment has not been fully embodied in the virtual models. Lots of research studies have explored the mechanism where physical entities interact with their environment in reality. However, there is still an urgent need on their corresponding digital expression method, which will lead to an efficient and accurate prediction in the future DT.

\section{Conclusions}

After the concept of DT was proposed in 2003, DT has attracted an increasing amount of attention in both industry and academia. The DT model has also evolved from the original three-dimensional DT model to a more sophisticated five-dimensional DT model composed of a physical object, a virtual model, DT services, DT data and a connection. The majority of DT literature focuses on the conceptual development of DT frameworks 
for a specific implementation area. Hence, this paper summarized the enabling technologies to implement DT according to the different components in the DT model. Based on these technologies, this paper provided a comprehensive survey from two perspectives: (1) applications in four product-lifecycle phases, i.e. product design, manufacturing, operation and maintenance, and recycling; and (2) applications in four categorized engineering fields, including aerospace engineering, tunneling and underground engineering, wind engineering, and the IoT. Key enabling technologies, such as sensor technology, virtual modeling technologies, data processing technologies and data transmission methods, in different application scenarios are also categorized. At the same time, this paper has mentioned the shortcomings of the lack of environmental factors in the DT model and discusses the six-dimensional DT model concerning environment.

DT is an emerging technology with significant potential. However, the following limitations hinder the thriving of DT: (1) Most DT models contain only geometric models. Although some recent research considered physical models, there is still a lack of in behavioral modeling and rule modeling. (2) Delay and distortions occur in data transmission. Current data transmission methods fail to satisfy the demand of high accuracy and high speed due to the large amount of data that need to be transmitted simultaneously. (3) Current data analysis algorithms and methods need improvement in both accuracy and rapidity. (4) There are various DT platforms for different applications, especially in complex equipment monitoring and IoT fields. However, because of the diversity in communication protocols and different service demands, the development period of these platforms is too long to form a unified DT platform.

In response to these shortcomings, future research directions for DT should focus on the following. (1) The development of a data format that contains all of the information and shows uniformity with real-world data, including geometry, physical, behavioral and rules information. (2) The implementation of the latest communication method, such as 5G, to satisfy the demand of high accuracy and low latency. (3) The optimization of algorithms to improve the speed and accuracy of the algorithms. (4) The development of methods for integrating different communication protocols and data communication interfaces with various services to develop a unified DT platform.

\author{
Abbreviations \\ ADT Airframe Digital Twin \\ AFMS Automated Flow-Shop Manufacturing System \\ BOL Beginning of life \\ BIM Building Information Model \\ BRM Behavior and Rule Model \\ CART Classification and Regression Trees \\ CFD Computational Fluid Dynamics \\ CPMS Cyber Physical Manufacturing System \\ CPPS Cyber Physical Production System \\ CPS Cyber Physical System \\ DBN Dynamic Bayesian Network \\ DD Digital-twin Data \\ DDDAS Dynamic Data-driven Application System \\ DFM Data Fusion Model \\ DM Digital Model \\ DS Digital Shadow
}




$\begin{array}{lll}\text { JIMSE } & \text { DT } & \text { Digital Twin } \\ 2,1 & \text { DTPD } & \text { Digital Twin-driven Product Design } \\ \text { DTS } & \text { Digital Twin Shop-floor } \\ \text { EEE } & \text { Electrical and Electronic Equipment } \\ \text { FAL } & \text { Final Assembly Line } \\ \text { FEM } & \text { Finite Elements Model } \\ \text { GBR } & \text { Gradient Boosting Regressor } \\ \text { GSM } & \text { Geometric and Shape Model } \\ \text { HDFS } & \text { Hadoop Distributed File System } \\ \text { HDTM } & \text { Hierarchical Digital Twin Model } \\ \text { iDMU } & \text { Industrial Digital Mock-Up } \\ \text { IoT } & \text { Internet of Things } \\ \text { MAM } & \text { Manufacturing Attribute Model } \\ \text { MCPS } & \text { Manufacturing Cyber-physical System } \\ \text { MES } & \text { Manufacturing Execution System } \\ \text { MOL } & \text { Middle-of-life } \\ \text { NASA } & \text { National Aeronautics and Space Administration } \\ \text { NBT } & \text { Noise Barrier Tunnel } \\ \text { OM } & \text { Operation \& Maintenance } \\ \text { PCT } & \text { Principal Component Transform } \\ \text { PDM } & \text { Product Definition Model } \\ \text { PE } & \text { Physical Entity } \\ \text { PHM } & \text { Prognostics and Health Managementl } \\ \text { PLM } & \text { Production Lifecycle Management } \\ \text { PMDT } & \text { Product Manufacturing Digital Twin } \\ \text { PS } & \text { Physical Shop Floor } \\ \text { PSO } & \text { Particle Swarm Optimization } \\ \text { QR } & \text { Quick Response } \\ \text { RDT } & \text { Reconfigure Digital Twin } \\ \text { RDTMS } & \text { Reconfigurable Digital Twin Manufacturing System } \\ \text { RFID } & \text { Radio Frequency Identification } \\ \text { RFR } & \text { Random Forest Regressor } \\ \text { RMS } & \text { Reconfigurable Manufacturing System } \\ \text { RUL } & \text { Remaining Useful Life } \\ \text { SDTD } & \text { Shop-floor Digital Twin Data } \\ \text { SMA } & \text { Shape Memory Alloy } \\ \text { SME } & \text { Medium-sized Enterprises } \\ \text { SP } & \text { Smart Production } \\ \text { Ss } & \text { Services } \\ \text { SSS } & \text { Shop-floor Service System } \\ \text { SVRS } & \text { Support Vector Regressor } \\ \text { UHVCS } & \text { Ultra-high-Voltage Converter Station } \\ \text { UIDs } & \text { Unique Identifiers } \\ \text { UWB } & \text { Ultra-wide Band } \\ \text { VE } & \text { Virtual Equipment } \\ \text { VS } & \text { Virtual Shop-floor } \\ \text { WEEE } & \text { Waste Electrical and Electronic Equipment } \\ \text { WT } & \text { Wind Turbine } \\ & \\ & & \\ & & \end{array}$




\section{References}

Abramovici, M., Gobel, J.C. and Dang, H.B. (2016), "Semantic data management for the development and continuous reconfiguration of smart products and systems", Cirp Annals-Manufacturing Technology, Vol. 65, pp. 185-188.

Aivaliotis, P., Georgoulias, K., Arkouli, Z. and Makris, S. (2019), "Methodology for enabling DT using advanced physics-based modelling in predictive maintenance", Procedia Cirp, Vol. 81, pp. 417-422.

Alam, K.M. and Saddik, A.E. (2017), "C2ps: a DT architecture reference model for the cloud-based cyber-physical systems", IEEE Access, Vol. 5, pp. 2050-2062.

Atorf, L., Schorn, C., Rossmann, J. and Schlette, C. (2017), "A framework for simulation-based optimization demonstrated on reconfigurable robot Workcells", 2017 IEEE International Systems Engineering Symposium, Vienna Marriott Hotel, Vienna, October 11-13, 2017.

Balakrishnan, P., Ramesh Babu, K., Damodaran Naiju, C. and Madiajagan, M. (2019), "Design and implementation of DT for predicting failures in automobiles using machine learning algorithms", SAE Technical Paper, Vol. 28, pp. 159-165.

Barrie, D. and Kirk-Davidoff, D.J.A.C. (2010), "Physics weather response to a large wind turbine array", Atmospheric Chemistry and Physics, Vol. 10, pp. 769-775.

Bazilevs, Y., Deng, X., Korobenko, A., Lanza Di Scalea, F., Todd, M. and Taylor, S. (2015), "Isogeometric fatigue damage prediction in large-scale composite structures driven by dynamic sensor data", Journal of Applied Mechanics, Vol. 82, pp. 091008-091019.

Beer, G. (2003), Numerical Simulation in Tunnelling, Springer Science and Business Media, Secaucus, NJ.

Bhupathiraju, V. and Ravuri, R.P. (2014), "The dawn of big data - HBase", 2014 Conference on it in Business, Industry and Government (Csibig), Sri Aurobindo Institute Of Technology, Near Mr-10 Crossing, Indore-Ujjain Highway, Indore, MP, March 8-9, 2014.

Bielefeldt, B., Hochhalter, J. and Hartl, D. (2015), "Computationally efficient analysis of SMA sensory particles embedded in complex aerostructures using a substructure approach", ASME 2015 Conference on Smart Materials, Adaptive Structures and Intelligent Systems, American Society of Mechanical Engineers Digital Collection, Colorado Springs, September 21-23, 2015.

Boschert, S., Heinrich, C. and Rosen, R. (2018), "Next generation DT", Proceedings of TMCE Las Palmas De Gran Canaria.

Castelli, G., Cesta, A., Diez, M., Padula, M., Ravazzani, P., Rinaldi, G., Savazzi, S., Spagnuolo, M., Strambini, L., Tognola, G. and Campana, E.F. (2019), "Urban intelligence: a modular, fully integrated, and evolving model for cities DTning", 2019 IEEE 16th International Conference on Smart Cities: Improving Quality of Life Using ICT and IoT and AI (Honet-ICT), University Of North Carolina at Charlotte, October 6-9, 2019, Charlotte, NC.

Chen, L.T., Poulos, H., Loganathan, N.J.J.O.G. and Engineering, G. (1999), "Pile responses caused by tunneling”, Journal of Geotechnical and Geoenvironmental Engineering, Vol. 125, pp. 207-215.

Cheng, J., Chen, W., Tao, F. and Lin, C.-L. (2018), "Industrial IoT in $5 \mathrm{~g}$ environment towards smart manufacturing", Journal of Industrial Information Integration, Vol. 10, pp. 10-19.

Chhetri, S.R., Faezi, S., Canedo, A. and Al Faruque, M.A. (2019), Quilt: Quality Inference from Living DTs in IoT-Enabled Manufacturing Systems, Assoc Computing Machinery, New York.

Ciavotta, M., Alge, M., Menato, S., Rovere, D. and Pedrazzoli, P.J.P.M. (2017), "A microservice-based middleware for the digital factory", Procedia Manufacturing, Vol. 11, pp. 931-938.

Coronado, P.D.U., Lynn, R., Louhichi, W., Parto, M., Wescoat, E. and Kurfess, T. (2018), "Part data integration in the shop floor DT: mobile and cloud technologies to enable a manufacturing execution system", Journal of Manufacturing Systems, Vol. 48, pp. 25-33.

Dröder, K., Bobka, P., Germann, T., Gabriel, F. and Dietrich, F. (2018), "A machine learning-enhanced DT approach for human-robot-collaboration”, Procedia CIRP, Vol. 76, pp. 187-192. 
JIMSE 2,1

Eliane Fourgeau, E.G., Adli, H., Fernandes, C. and Hagege, M. (2016), "System engineering Workbench for multi-views systems methodology with $3 \mathrm{~d}$ experience platform. The aircraft radar use case", Complex Systems Design and Management Asia, Vol. 426, pp. 269-270.

Erikstad, S.O. (2017), "Merging physics, big data analytics and simulation for the next-generation DTs", High-Performance Marine Vehicles, pp. 141-151.

Ferguson, S., Bennett, E. and Ivashchenko, A. (2017), "DT tackles design challenges", World Pumps, Vol. 2017, pp. 26-28.

Finno, R.J. and Clough, G.W.J.J.O.G.E. (1985), "Evaluation of soil response to EPB shield tunneling", Journal of Geotechnical Engineering, Vol. 111, pp. 155-173.

Fiore, G. and Selig, M.S. (2014), “A simulation of operational damage for wind turbine blades”, 32nd AIAA Applied Aerodynamics Conference, Atlanta, GA, June 16-20, 2014.

Fuller, A., Fan, Z., Day, C. and Barlow, C. (2019), "DT: enabling technology, challenges and open research", IEEE Access, Vol. 8, pp. 108952-108971.

Future MR (2019), "DT market is estimated to grow at a CAGR of 42.54\% from 2018 - 2025", available at: https://www.marketresearchfuture.com/press-release/digital-twin-market.

Gao, R., Wang, L., Teti, R., Dornfeld, D., Kumara, S., Mori, M. and Helu, M. (2015), "Cloud-enabled prognosis for manufacturing", CIRP Annals, Vol. 64, pp. 749-772.

Ge (2020a), "Building a DT, bolstering the power of a wind turbine", available at: https://www.ge.com/ renewableenergy/stories/improving-wind-power-with-digital-twin-turbines.

Ge (2020b), "DT”, available at: https:/www.ge.com/digital/applications/digital-twin.

Ghobadi, M. (2017), "Windgemini DT for wind turbine operations”, available at: https://www.dnvgl. com/news/new-windgemini-advances-wind-turbine-operations-104699.

Glaessgen, E. and Stargel, D. (2012), "The DT paradigm for future NASA and U.S. Air force vehicles", AIAA/ASME/ASCE/AHS/ASC Structures, Structural Dynamics and Materials Conference AIAA/ASME/AHS Adaptive Structures Conference AIAA, Honolulu, April 23-26, 2012.

Gockel, B., Tudor, A., Brandyberry, M., Penmetsa, R. and Tuegel, E. (2012), "Challenges with structural life forecasting using realistic mission profiles", 53rd AIAA/ASME/ASCE/AHS/ASC Structures, Structural Dynamics and Materials Conference 20th AIAA/ASME/AHS Adaptive Structures Conference 14th AIAA, Honolulu, April 23-26, 2012.

Google Scholar (2020), available at: https://scholar.google.com/scholar?as_q=\&as_epq=digital+ twins\&as_oq $=\& a s \_e q=\& a s \_o c c t=$ title\&as_sauthors $=\& a s \_p u b l i c a t i o n=\& a s \_y l o=2019 \&$ as_yhi $=2019 \& h l=$ en\&as_sdt $=1 \% 2 c 5 \& a s \_v i s=1$.

Grieves, M. (2014), “DT: manufacturing excellence through virtual factory replication”, available at: https://www.researchgate.net/publication/275211047_Digital_Twin_Manufacturing_ Excellence_through_Virtual_Factory_Replication/citation/download.

Grieves, M. and Vickers, J. (2017), "DT: mitigating unpredictable, undesirable emergent behaviro in complex systems", in Al, F.J.K.E. (Ed.), Transdisciplinary Perspectives on Complex Systems, Springer International Publishing.

Hochhalter, J., Leser, W.P., Newman, J.A., Gupta, V.K., Yamakov, V., Cornell, S.R., Willard, S.A. and Heber, G. (2014), Coupling Damage-Sensing Particles to the Digitial Twin Concept, National Aeronautics and Space Administration, Langley Research Center, Hampton, VA.

Hu, W., Chen, W., Wang, X., Jiang, Z., Wang, Y., Verma, A.S. and Teuwen, J.J.E. (2021), "A computational framework for coating fatigue analysis of wind turbine blades due to rain erosion”, Renewable Energy, Vol. 170, pp. 236-250.

Kapil, S., Chawla, M. and Ansari, M.D. (2016), "On K-means data clustering algorithm with genetic algorithm”, 2016 Fourth International Conference on Parallel, Distributed and Grid Computing (PDGC), Jaypee University Of Information Technology, Waknaghat, Solan, December 2224, 2016. 
Kim, J. and Kim, S.A. (2020), "Lifespan prediction technique for DT-based noise barrier tunnels", Sustainability, Vol. 12, p. 14.

Koren, Y., Gu, X. and Guo, W. (2018), "Reconfigurable manufacturing systems: principles, design, and future trends", Frontiers of Mechanical Engineering, Vol. 13, pp. 121-136.

Kritzinger, W., Karner, M., Traar, G., Henjes, J. and Sihn, W. (2018), "DT in manufacturing: a categorical literature review and classification", IFAC-Papersonline, Vol. 51, pp. 1016-1022.

Lee, J., Bagheri, B. and Kao, H.-A. (2015), "A cyber-physical systems architecture for industry 4.0based manufacturing systems", Manufacturing Letters, Vol. 3, pp. 18-23.

Leng, J., Zhang, H., Yan, D., Liu, Q., Chen, X. and Zhang, D. (2019), "DT-driven manufacturing cyberphysical system for parallel controlling of smart workshop", Journal of Ambient Intelligence and Humanized Computing, Vol. 10, pp. 1155-1166.

Li, C., Mahadevan, S., Ling, Y., Choze, S. and Wang, L. (2017), "Dynamic Bayesian network for aircraft wing health monitoring DT", AIAA Journal, Vol. 55, pp. 930-941.

Liao, S.-M., Liu, J.-H., Wang, R.-L., Li, Z.-M.J.T. and Technology, U.S. (2009), "Shield tunneling and environment protection in Shanghai soft ground", Tunnelling and Underground Space Technology, Vol. 24, pp. 454-465.

Liau, Y., Lee, H. and Ryu, K. (2017), "DT concept for smart injection molding", 2017 The 5th International Conference on Mechanical Engineering, Materials Science and Civil Engineering IOP Publishing, Kuala Lumpur, December 15-16, 2017.

Liu, Z., Meyendorf, N. and Mrad, N. (2018), "The role of data fusion in predictive maintenance using DT", in Chimenti, D.E. and Bond, L.J. (Eds), 44th Annual Review of Progress in Quantitative Nondestructive Evaluation, Amer Inst Physics, Melville, Vol. 37.

Liu, Q., Zhang, H., Leng, J. and Chen, X. (2019a), "DT-driven rapid Individualised designing of automated flow-shop manufacturing system", International Journal of Production Research, Vol. 57, pp. 3903-3919.

Liu, Y., Zhang, L., Yang, Y., Zhou, L., Ren, L., Wang, F., Liu, R., Pang, Z. and Deen, M.J. (2019b), “A novel cloud-based framework for the elderly healthcare services using DT", IEEE Access, Vol. 7, pp. 49088-49101.

Madni, A.M., Madni, C.C. and Lucero, S. (2019), "Leveraging DT technology in model-based systems engineering", System, Vol. 7, p. 7.

Magargle, R., Johnson, L., Mandloi, P., Davoudabadi, P., Kesarkar, O., Krishnaswamy, S., Batteh, J. and Pitchaikani, A. (2017), "A simulation-based DT for model-driven health monitoring and predictive maintenance of an automotive braking system", 12th International Modelica Conference, Linköping University Electronic Press, Prague, May 15-17, 2017.

Malik, A.A. and Bilberg, A. (2018), "DTs of human robot collaboration in a production setting", Procedia Manufacturing, Vol. 17, pp. 278-285.

Mas, F., Menéndez, J.L., Oliva, M., Gómez, A. and Ríos, J. (2013), “Collaborative engineering paradigm applied to the aerospace industry", IFIP International Conference on Product Lifecycle Management, Nantes, July 8-10, 2013.

Mas, F., Menéndez, J.L., Oliva, M., Ríos, J. and Olmos, V. (2014), "IDMU as the collaborative engineering engine: research experiences in Airbus", International ICE Conference on Engineering, Technology and Innovation, Bergamo, June 23-25, 2014.

Miskinis, C. (2019), "The history and creation of the DT concept", available at: https://www.challenge. org/insights/digital-twin-history/.

Mohammadi, N. and Taylor, J.E. (2017), "Smart city DTs", 2017 IEEE Symposium Series on Computational Intelligence (SSCI), Honolulu, November 27 - December 1, 2017.

Neanex (2020), "Digital tunnel twins", available at: https://www.neanex.com/en/cases/digitaltunnel-twins. 
JIMSE 2,1

Negri, E., Fumagalli, L. and Macchi, M. (2017), "A review of the roles of DT in cps-based production systems”, Procedia Manufacturing, Vol. 11, pp. 939-948.

Noda, M. and Flay, R. (1999), "A simulation model for wind turbine blade fatigue loads", Journal of Wind Engineering Industrial Aerodynamics, Vol. 83, pp. 527-540.

Noel, N. and Jackson, K. (2015), "Ge launches the next evolution of wind energy making renewables more efficient, economic: the digital wind farm", available at: https://www.ge.com/news/pressreleases/ge-launches-next-evolution-wind-energy-making-renewables-more-efficient-economic.

Ongo, G. and Kusuma, G.P. (2018), "Hybrid database system of mysql and mongodb in Web application development", 2018 International Conference on Information Management and Technology (Icimtech), Bina Nusantara University, Jakarta, September 3-5, 2018.

Panetta, K. (2016), “Gartner's top 10 strategic technology trends for 2017", available at: https://www. gartner.com/smarterwithgartner/gartners-top-10-technology-trends-2017/.

Panetta, K. (2017), “Gartner's top 10 strategic technology trends for 2018”, available at: https://www. gartner.com/smarterwithgartner/gartners-top-10-technology-trends-2018/.

Panetta, K. (2018), "Gartner's top 10 strategic technology trends for 2019”, available at: https://www. gartner.com/smarterwithgartner/gartners-top-10-technology-trends-2019/.

Parrott, A. and Warshaw, L. (2017), "Industry 4.0 and the DT", available at: https://www2.deloitte. com/us/en/insights/focus/industry-4-0/digital-twin-technology-smart-factory.html.

Priggemeyer, M. and Rossmann, J. (2018), "Simulation-based control of reconfigurable robotic Workcells: interactive planning and execution of processes in cyber-physical systems", ISR 2018; 50th International Symposium on Robotics", Vde, Munich, June 20-21, 2018.

Qamsane, Y., Chen, C.-Y., Balta, E.C., Kao, B.-C., Mohan, S., Moyne, J., Tilbury, D. and Barton, K. (2019), "A unified DT framework for real-time monitoring and evaluation of smart manufacturing systems", 2019 IEEE 15th International Conference on Automation Science and Engineering (Case), IEEE, University Of British Columbia, Vancouver, BC, August 2226, 2019.

Qi, Q., Tao, F., Zuo, Y. and Zhao, D. (2018), "DT service towards smart manufacturing”, Procedia CIRP, Vol. 72, pp. 237-242.

Qi, Q., Tao, F., Hu, T., Anwer, N., Liu, A., Wei, Y., Wang, L. and Nee, A. (2021), "Enabling technologies and tools for DT", Journal of Manufacturing Systems, Vol. 58, pp. 3-21.

Reifsnider, K. and Majumdar, P. (2013), "Multiphysics stimulated simulation DT methods for fleet management", 54th AIAA/ASME/ASCE/AHS/ASC Structures, Structural Dynamics, and Materials Conference, Boston, MA, Apirl 8-11, 2013.

Ríos, J., Hernández, J.C., Oliva, M. and Mas, F. (2015), "Product avatar as digital counterpart of a physical individual product: literature review and implications in an aircraft", Advances in Transdisciplinary Engineering, Vol. 2, pp. 657-666.

Ríos, J., Morate, F.M., Oliva, M. and Hernández, J.C. (2016), "Framework to support the aircraft digital counterpart concept with an industrial design view", International Journal of Agile Systems Management, Vol. 9, pp. 212-231.

Rosen, R., Von Wichert, G., Lo, G. and Bettenhausen, K.D. (2015), “About the importance of autonomy and DTs for the future of manufacturing", IFAC-Papersonline, Vol. 48, pp. 567-572.

Ruohomäki, T., Airaksinen, E., Huuska, P., Kesäniemi, O., Martikka, M. and Suomisto, J. (2018), "Smart city platform enabling DT", 2018 International Conference on Intelligent Systems (IS), Phuket, November 17-19, 2018.

Saddik, A.E. (2018), "DTs: the convergence of multimedia technologies", IEEE Multimedia, Vol. 25, pp. 87-92.

Schleich, B., Anwer, N., Mathieu, L. and Wartzack, S. (2017), "Shaping the DT for design and production engineering", CIRP Annals, Vol. 66, pp. 141-144. 
Schroeder, G.N., Steinmetz, C., Pereira, C.E. and Espindola, D.B. (2016), "DT data modeling with automationml and a communication methodology for data exchange", IFAC-Papersonline, Vol. 49, pp. 12-17.

Seshadri, B.R. and Krishnamurthy, T. (2017), "Structural health management of damaged aircraft structures using the DT concept", 25th AIAA/AHS Adaptive Structures Conference Grapevine, January 9-13, 2017.

Sivalingam, K., Sepulveda, M., Spring, M. and Davies, P. (2018), "A review and methodology development for remaining useful life prediction of offshore fixed and floating wind turbine power converter with DT technology perspective", 2018 2nd International Conference on Green Energy and Applications (ICGEA), IEEE, Singapore, March 24-26, 2018.

Söderberg, R., Wärmefjord, K., Carlson, J.S. and Lindkvist, L. (2017), "Toward a DT for real-time geometry assurance in individualized production”, Cirp Annals, Vol. 66, pp. 137-140.

Stark, R., Kind, S. and Neumeyer, S. (2017), "Innovations in digital modelling for next generation manufacturing system design", CIRP Annals, Vol. 66, pp. 169-172.

State Council Of China (2015), "Made in China 2025", available at: http://www.gov.cn/zhengce/content/ 2015-05/19/content_9784.htm.

Systems D (2020), "Delmia industrial engineering", available at: https://www.3ds.com/productsservices/delmia/disciplines/industrial-engineering/tag/5960-17402/.

Tao, F. and Zhang, M. (2017), "DT shop-floor: a new shop-floor paradigm towards smart manufacturing", IEEE Access, Vol. 5, pp. 20418-20427.

Tao, F., Cheng, J. and Qi, Q. (2017a), "IIHUB: an industrial Internet-of-things Hub toward smart manufacturing based on cyber-physical system", IEEE Transactions on Industrial Informatics, Vol. 14, pp. 2271-2280.

Tao, F., Cheng, Y., Cheng, J., Zhang, M., Xu, W. and Qi, Q. (2017b), "Theories and technologies for cyber-physical fusion in DT shop-floor", Computer Integrated Manufacturing Systems, Vol. 23, pp. 1603-1611.

Tao, F., Cheng, J., Qi, Q., Zhang, M., Zhang, H. and Sui, F. (2018a), "DT-driven product design, manufacturing and service with big data", The International Journal of Advanced Manufacturing Technology, Vol. 94, pp. 3563-3576.

Tao, F., Qi, Q., Liu, A. and Kusiak, A. (2018b), "Data-driven smart manufacturing", Journal of Manufacturing Systems, Vol. 48, pp. 157-169.

Tao, F., Zhang, H., Liu, A. and Nee, A.Y. (2018c), "DT in industry: state-of-the-art", IEEE Transactions on Industrial Informatics, Vol. 15, pp. 2405-2415.

Tao, F., Zhang, M., Liu, Y. and Nee, A. (2018d), "DT driven prognostics and health management for complex equipment", CIRP Annals, Vol. 67, pp. 169-172.

Tao, F., Sui, F., Liu, A., Qi, Q., Zhang, M., Song, B., Guo, Z., Lu, S.C.-Y. and Nee, A. (2019), "DT-driven product design framework", International Journal of Production Research, Vol. 57, pp. 3935-3953.

Tuegel, E. (2012), "The airframe DT: some challenges to realization", 53rd AIAA/ASME/ASCE/ AHS/ASC Structures, Structural Dynamics and Materials Conference, Honolulu, April 2326, 2012.

Tuegel, E.J., Ingraffea, A.R., Eason, T.G. and Spottswood, S.M. (2011a), "Reengineering aircraft structural life prediction using a DT", International Journal of Aerospace Engineering, Vol. 2011, p. 14.

Tuegel, E.J., Ingraffea, A.R., Eason, T.G. and Spottswood, S.M. (2011b), "Reengineering aircraft structural life prediction using a DT", International Journal of Aerospace Engineering, Vol. 2011.

Tunnelware (2020), “Tunnel boring 4.0”, available at: https://www.tunnelware.io/.

Uhlemann, T.H.J., Lehmann, C. and Steinhilper, R. (2017), "The DT: realizing the cyber-physical production system for industry 4.0", Procedia CIRP, Vol. 61, pp. 335-340. 
JIMSE 2,1

34

Verner, I., Cuperman, D., Fang, A., Reitman, M., Romm, T. and Balikin, G. (2018), Robot Online Learning through DT Experiments: A Weightlifting Project, Springer International Publishing, Cham.

Vrabic, R., Erkoyuncu, J.A., Butala, P. and Roy, R. (2018), "DTs: understanding the added value of integrated models for through-life engineering services", Procedia Manufacturing, Vol. 16, pp. 139-146.

Wang, X.V. and Wang, L. (2019), "DT-based WEEE recycling, recovery and remanufacturing in the background of industry 4.0", International Journal of Production Research, Vol. 57, pp. 3892-3902.

Wang, X.V., Kemény, Z., Váncza, J. and Wang, L. (2017), "Human-robot collaborative assembly in cyber-physical production: classification framework and implementation", CIRP Annals, Vol. 66, pp. 5-8.

Wang, J., Ye, L., Gao, R.X., Li, C. and Zhang, L. (2019), "DT for rotating machinery fault diagnosis in smart manufacturing", International Journal of Production Research, Vol. 57, pp. 3920-3934.

Wikipedia (2020), "Internet of Things - Wikipedia", available at: https:/en.wikipedia.org/wiki/ internet_of_things.

Zaccaria, V., Stenfelt, M., Aslanidou, I. and Kyprianidis, K.G. (2018), "Fleet monitoring and diagnostics framework based on DT of aero-engines", ASME Turbo Expo 2018: Turbomachinery Technical Conference and Exposition, American Society of Mechanical Engineers Digital Collection, Oslo, June 11-15, 2018.

Zambal, S., Eitzinger, C., Clarke, M., Klintworth, J. and Mechin, P.-Y. (2018), "A DT for composite parts manufacturing: effects of defects analysis based on manufacturing data", 2018 IEEE 16th International Conference on Industrial Informatics (Indin), IEEE, Porto, July 18-20, 2018.

Zhang, H., Liu, Q., Chen, X., Zhang, D. and Leng, J. (2017), “A DT-based approach for designing and multi-objective optimization of Hollow glass production line", IEEE Access, Vol. 5, pp. 26901-26911.

Zhang, C., Xu, W., Liu, J., Liu, Z., Zhou, Z. and Pham, D.T. (2019a), "DT-enabled reconfigurable modeling for smart manufacturing systems", International Journal of Computer Integrated Manufacturing, pp. 1-25.

Zhang, C., Xu, W., Liu, J., Liu, Z., Zhou, Z. and Pham, D.T. (2019b), "A reconfigurable modeling approach for DT-based manufacturing system”, Procedia CIRP, Vol. 83, pp. 118-125.

Zhang, H., Zhang, G. and Yan, Q. (2019c), "DT-driven cyber-physical production system towards smart shop-floor", Journal of Ambient Intelligence and Humanized Computing, Vol. 10, pp. 4439-4453.

Zheng, P., Lin, T., Chen, C. and Xu, X. (2018), "A systematic design approach for service Innovation of smart product-service systems", Journal of Cleaner Production, Vol. 201, pp. 657-667.

Zhuang, C., Liu, J. and Xiong, H. (2018), "DT-based smart production management and control framework for the complex product assembly shop-floor", The International Journal of Advanced Manufacturing Technology, Vol. 96, pp. 1149-1163.

\section{Corresponding author}

Weifei Hu can be contacted at: weifeihu@zju.edu.cn

For instructions on how to order reprints of this article, please visit our website:

www.emeraldgrouppublishing.com/licensing/reprints.htm

Or contact us for further details: permissions@emeraldinsight.com 